\title{
Assembly Pattern of Supramolecular Hydrogel Induced by Lower Critical Solution Temperature Behavior of Low-Molecular-Weight Gelator
}

Shuanggen $\mathrm{Wu},{ }^{\mathrm{a}}$ Qiao Zhang, ${ }^{\mathrm{a}}$ Yan Deng, ${ }^{a}$ Xing Li, ${ }^{a}$ Zheng Luo, ${ }^{\mathrm{a}}$ Bo Zheng, ${ }^{\mathrm{b} *}$ and Shengyi Donga*

${ }^{1}$ College of Chemistry and Chemical Engineering, Hunan University, 410082, Changsha, P. R. China.

${ }^{2}$ Key Laboratory of Synthetic and Natural Functional Molecule Chemistry of the Ministry of Education, College of Chemistry and Materials Science, Northwest University, Xi'an 710069, P. R. China.

*Corresponding author. Email: dongsy@hnu.edu.cn; zhengbo@nwu.edu.cn

\section{Supporting Information}

\section{Table of contents}

1. Materials and methods

2. Monomer preparation

3. Cycling tests of MF

4. Temperature-dependent UV/Vis of $\mathbf{M F}$

5. Rheology measurements of MF

6. Scanning electron microscopy of $\mathbf{M F}$

7. Transmission electron microscopy of $\mathbf{M F}$

8. Concentration-dependent ${ }^{1} \mathrm{H}$ NMR of $\mathbf{M F}$

9. Simulated chemical strucuture of $\mathbf{M F}$

10. Time-dependent ${ }^{1} \mathrm{H}$ NMR of $\mathbf{M F}$

11. Temperature-dependent ${ }^{1} \mathrm{H}$ NMR of $\mathbf{M F}$

12. Time-/concentration-/temperature-dependent ${ }^{1} \mathrm{H}$ NMR of $\mathbf{M H}$

13. Concentration of the sol part of MF hydrogel

14. Reference 


\section{Materials and methods}

All materials and reagents were commercially available and used without further purification. B21C7-CH2-NH2 and TC were prepared by reported methods [1]. ${ }^{1} \mathrm{H}$ NMR spectra were collected on a Varian Unity INOVA-400 or Bruker-AV400 with TMS or residual solvent as the internal standard. ${ }^{13} \mathrm{C}$ NMR spectra were recorded on a Bruker-AV400 spectrometer at $100 \mathrm{MHz}$. MALDI-TOF mass spectrometry was performed on a Shimadzu Biotech AXIMA Performance instrument. Temperature-dependent ${ }^{1} \mathrm{H}$ NMR spectra were collected by Bruker Ascend TM 400 MHz. UV/Vis spectra were recorded by a Shimadzu UV2600 spectrometer. Dynamic light scattering (DLS) was recorded by Zetasizer Nano ZSP. Rheologic performance was conducted by Anton Paar MCR 92. The laminator model PP15 was chosen with a diameter of $15 \mathrm{~mm}$ with the gap at $1 \mathrm{~mm}$. Images of the transmission electron microscope (TEM) and the scanning electron microscope (SEM) were collected on TecnaiG2 Spirit TWIN and Hitachi S-4800 or SU8010, respectively. Unless otherwise stated, samples were dissolved in Milli-Q water. Molecular structures were obtained by using Gaussian 09 software and DFT method with B3LYP functional. Cryo-TEM was carried out on a FEI Talos F200C (ThermoFisher).

\section{Monomer preparation}<smiles>O=C(O)c1c(F)c(F)c(F)c(F)c1C(=O)O</smiles><smiles>O=C(Cl)c1c(F)c(F)c(F)c(C(=O)Cl)c1F</smiles>

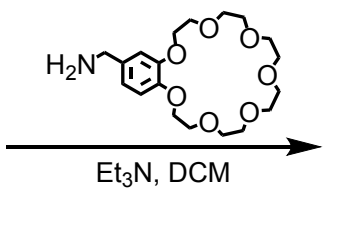<smiles>[2H]NC(=O)c1c(F)c(F)c(F)c(F)c1C(=O)N[2H]</smiles>

OF<smiles>O=C(O)c1c(F)c(F)c(C(=O)O)c(F)c1F</smiles><smiles>CCCS(=O)(=O)Cl</smiles><smiles>O=C(Cl)c1c(F)c(F)c(F)c(C(=O)Cl)c1F</smiles>

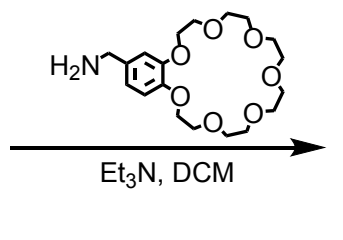<smiles>[R]NC(=O)c1c(F)c(F)c(F)c(C(=O)NC(F)(F)F)c1F</smiles><smiles>O=C(O)c1c(F)c(F)c(C(=O)O)c(F)c1F</smiles><smiles></smiles><smiles>CC(=O)c1c(F)c(F)c(C(=O)Cl)c(F)c1F</smiles>

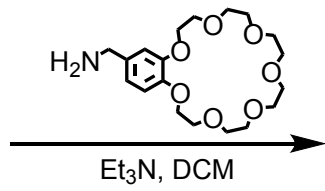<smiles>[R]NC(=O)c1c(F)c(F)c(C(=O)N[R])c(F)c1F</smiles>

PF<smiles>NCc1ccc2c(c1)COCCOCOCCOCCOC2</smiles>

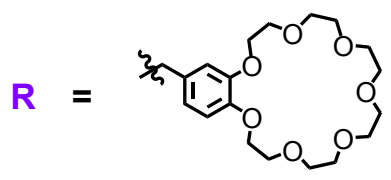

Scheme S1. Synthesis of OF, MF, and PF. 
Intuitively, PF is the best candidate among the ortho-, meta-, and para-substituted monomers; however, it is completely insoluble. OF has a low solubility in water $(<$ $0.1 \mathrm{mg} / \mathrm{mL}$, Figure S2). Unexpectedly, MF is the only water-soluble LMWG. The differences in solubility are ascribed to the substitution position. OF with two B21C7 units in the ortho-position has relatively small water accessible surfaces area (ASA). $\mathbf{P F}$, however, favors the formation of one-dimensional aggregates that easily expel water molecules, leading to insolubility in water. MF achieves a balance between the solubility (hydration effect) and aggregation (gelation process); hence, we focused on the LMWG MF and its LCST-induced gela-tion process.

OF: To a schlenk flask equipped a magnetic stirring bar, 3,4,5,6-tetrafluorophthalic acid $(0.339 \mathrm{~g}, 1.42 \mathrm{mmol})$ and thionyl chloride $(30 \mathrm{~mL})$ were added. The resulting mixture was stirred under reflux for $48 \mathrm{~h}$. After the reaction mixture was cooled to room temperature, extra thionyl chloride was removed under reduced pressure to give 3,4,5,6-tetrafluorophthaloyl dichloride, which was used for the following reaction without purification. B21C7-CH2-NH2 (1.10 g, $2.84 \mathrm{mmol})$ was added to a solution containing 3,4,5,6-tetrafluorophthaloyl dichloride in $\mathrm{CH}_{2} \mathrm{Cl}_{2} \quad(50 \mathrm{~mL})$ and triethylamine $(4 \mathrm{~mL})$. The resulting mixture was stirred at $25^{\circ} \mathrm{C}$ for $36 \mathrm{~h}$. When the reaction was complete, $\mathrm{HCl}$ was added to the reaction mixture and stirred at room temperature. After liquid separation, organic solvents were removed to obtain product OF as a white solid (1.15 g, 83\%); Melting point: $117.3-118.2{ }^{\circ} \mathrm{C}$. ${ }^{1} \mathrm{H}$ NMR (400 $\mathrm{MHz}, \mathrm{CDCl}_{3}$, room temperature) $\delta[\mathrm{ppm}]=7.17(\mathrm{~s}, 2 \mathrm{H}), 6.94(\mathrm{~s}, 2 \mathrm{H}), 6.73(\mathrm{~s}, 4 \mathrm{H})$, $4.41-4.40(\mathrm{~d}, J=4 \mathrm{~Hz}, 4 \mathrm{H}), 4.16(\mathrm{~s}, 4 \mathrm{H}), 4.08(\mathrm{~s}, 4 \mathrm{H}), 3.87(\mathrm{~m}, 8 \mathrm{H}), 3.84(\mathrm{~m}, 8 \mathrm{H})$, $3.66(\mathrm{~m}, 8 \mathrm{H}), 3.61(\mathrm{~m}, 16 \mathrm{H}) ;{ }^{13} \mathrm{C} \mathrm{NMR}\left(100 \mathrm{MHz}, \mathrm{CDCl}_{3}\right.$, room temperature) $\delta$ $[\mathrm{ppm}]=163.07,161.18,148.62,147.77,130.96,120.64,113.94,69.84,69.73,68.79$, 68.55, 43.64; ESI-TOF-HRMS: calcd. for $\left[\mathrm{M}+\mathrm{H}_{2} \mathrm{O}+\mathrm{H}\right]^{+}\left(\mathrm{C}_{46} \mathrm{H}_{63} \mathrm{~F}_{4} \mathrm{~N}_{2} \mathrm{O}_{17}\right) \mathrm{m} / \mathrm{z}$ 991.4057, found 991.4045, error $1.2 \mathrm{ppm}$.

MF: MF was prepared using the same procedure and obtained as a white solid. Yield: 83\%; Melting point: $124.4-125.9^{\circ} \mathrm{C} .{ }^{1} \mathrm{H}$ NMR (400 $\mathrm{MHz}, \mathrm{CDCl}_{3}$, room temperature) $\delta[\mathrm{ppm}]=6.85(\mathrm{~s}, 2 \mathrm{H}), 6.82(\mathrm{~s}, 4 \mathrm{H}), 6.73(\mathrm{~s}, 2 \mathrm{H}), 4.51-4.50(\mathrm{~d}, J=4 \mathrm{~Hz}, 4 \mathrm{H}), 4.13$ $(\mathrm{m}, 8 \mathrm{H}), 3.89(\mathrm{~m}, 8 \mathrm{H}), 3.75(\mathrm{~m}, 8 \mathrm{H}), 3.70(\mathrm{~m}, 8 \mathrm{H}), 3.64(\mathrm{~m}, 16 \mathrm{H}) ;{ }^{13} \mathrm{C}$ NMR $(100$ $\mathrm{MHz}, \mathrm{CDCl}_{3}$, room temperature) $\delta[\mathrm{ppm}]=157.86,149.01,148.33,130.29,120.60$, $114.41,113.88,70.93,70.39,69.73,69.19,43.87$; ESI-TOF-HRMS: calcd. for [M+ $\mathrm{Na}]^{+}\left(\mathrm{C}_{46} \mathrm{H}_{60} \mathrm{~F}_{4} \mathrm{~N}_{2} \mathrm{NaO}_{16}\right) \mathrm{m} / \mathrm{z}$ 995.3771, found 995.3807 , error $3.6 \mathrm{ppm}$.

PF: PF was prepared using the same procedure and obtained as a white solid. Yield: 96\%; Melting point: $122.5-124.0{ }^{\circ} \mathrm{C} .{ }^{1} \mathrm{H}$ NMR (400 MHz, $\mathrm{CDCl}_{3}$, room temperature) $\delta[\mathrm{ppm}]=7.44(\mathrm{~s}, 2 \mathrm{H}), 6.80(\mathrm{~s}, 2 \mathrm{H}), 6.78(\mathrm{~s}, 4 \mathrm{H}), 4.40-4.39(\mathrm{~d}, J=8 \mathrm{~Hz}, 4 \mathrm{H}), 4.08$ $(\mathrm{m}, 8 \mathrm{H}), 3.85(\mathrm{~m}, 8 \mathrm{H}), 3.72(\mathrm{~m}, 8 \mathrm{H}), 3.67(\mathrm{~m}, 8 \mathrm{H}), 3.60(\mathrm{~m}, 16 \mathrm{H}) ;{ }^{13} \mathrm{C}$ NMR $(100$ $\mathrm{MHz}, \mathrm{CDCl}_{3}$, room temperature) $\delta[\mathrm{ppm}]=157.73,149.05,148.27,130.35,120.58$, 114.31, 113.51, 70.98, 70.49, 69.73, 69.32, 69.06, 43.70; ESI-TOF-HRMS: calcd. for $[\mathrm{M}+\mathrm{Na}]^{+}\left(\mathrm{C}_{46} \mathrm{H}_{60} \mathrm{~F}_{4} \mathrm{~N}_{2} \mathrm{NaO}_{16}\right) \mathrm{m} / \mathrm{z}$ 995.3771, found 995.3810 , error $3.9 \mathrm{ppm}$. 
MH: MH was prepared using the same procedure and obtained as a yellow solid (which is highly hydroscopic and become a viscous glue-like liquid). Yield: $76 \%$. ${ }^{1} \mathrm{H}$ NMR (400 MHz, $\mathrm{CDCl}_{3}$, room temperature) $8.27(\mathrm{~s}, 1 \mathrm{H}), 7.93(\mathrm{~d}, J=8 \mathrm{~Hz}, 2 \mathrm{H}), 7.46$ (t, $J=8 \mathrm{~Hz}, 1 \mathrm{H}), 7.36(\mathrm{~s}, 2 \mathrm{H}), 6.78(\mathrm{~s}, 2 \mathrm{H}), 6.74(\mathrm{~d}, J=8 \mathrm{~Hz}, 2 \mathrm{H}), 6.69(\mathrm{~d}, J=8 \mathrm{~Hz}$, $2 \mathrm{H}), 4.40(\mathrm{~d}, J=4 \mathrm{~Hz}, 4 \mathrm{H}), 4.04(\mathrm{~s}, 8 \mathrm{H}), 3.82-3.85(\mathrm{~m}, 8 \mathrm{H}), 3.72-3.68(\mathrm{~m}, 16 \mathrm{H})$, $3.61(\mathrm{~s}, 16 \mathrm{H}) ;{ }^{13} \mathrm{C} \mathrm{NMR}\left(125 \mathrm{MHz}, \mathrm{CDCl}_{3}\right.$, room temperature) $\delta(\mathrm{ppm}): 166.54$, $148.84,148.14,134.24,131.36,130.43,129.00,125.26,120.86,114.06,114.02$, 71.02, 70.95, 70.91, 70.50, 70.45, 69.74, 69.11, 43.77; HRESIMS: calcd for $[\mathrm{M}+\mathrm{K}]^{+}$ $\left(\mathrm{C}_{46} \mathrm{H}_{64} \mathrm{KN}_{2} \mathrm{O}_{16}\right) \mathrm{m} / \mathrm{z}$ 939.3893, found 939.3896, error $0.3 \mathrm{ppm}$.

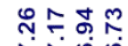

กิ่0

ก

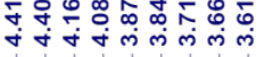

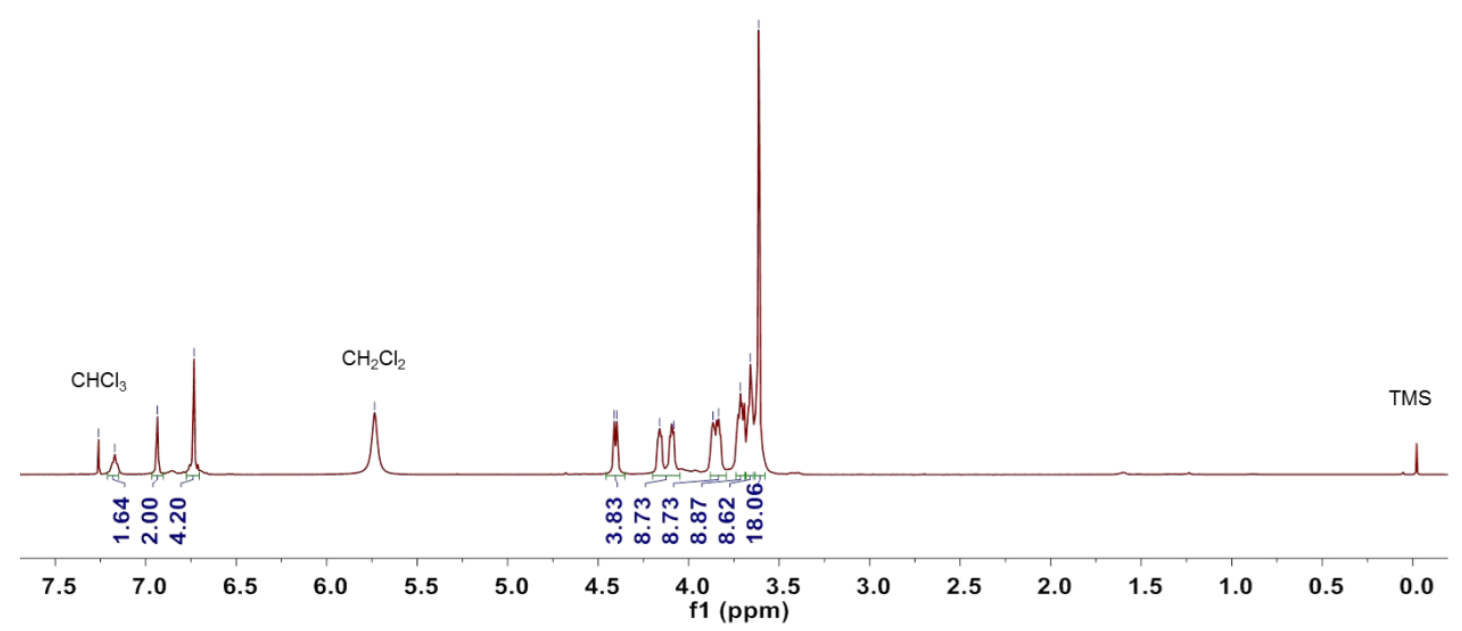

Figure S1. ${ }^{1} \mathrm{H}$ NMR spectrum $\left(400 \mathrm{MHz}, \mathrm{CDCl}_{3}\right.$, room temperature) of $\mathbf{O F}$. 


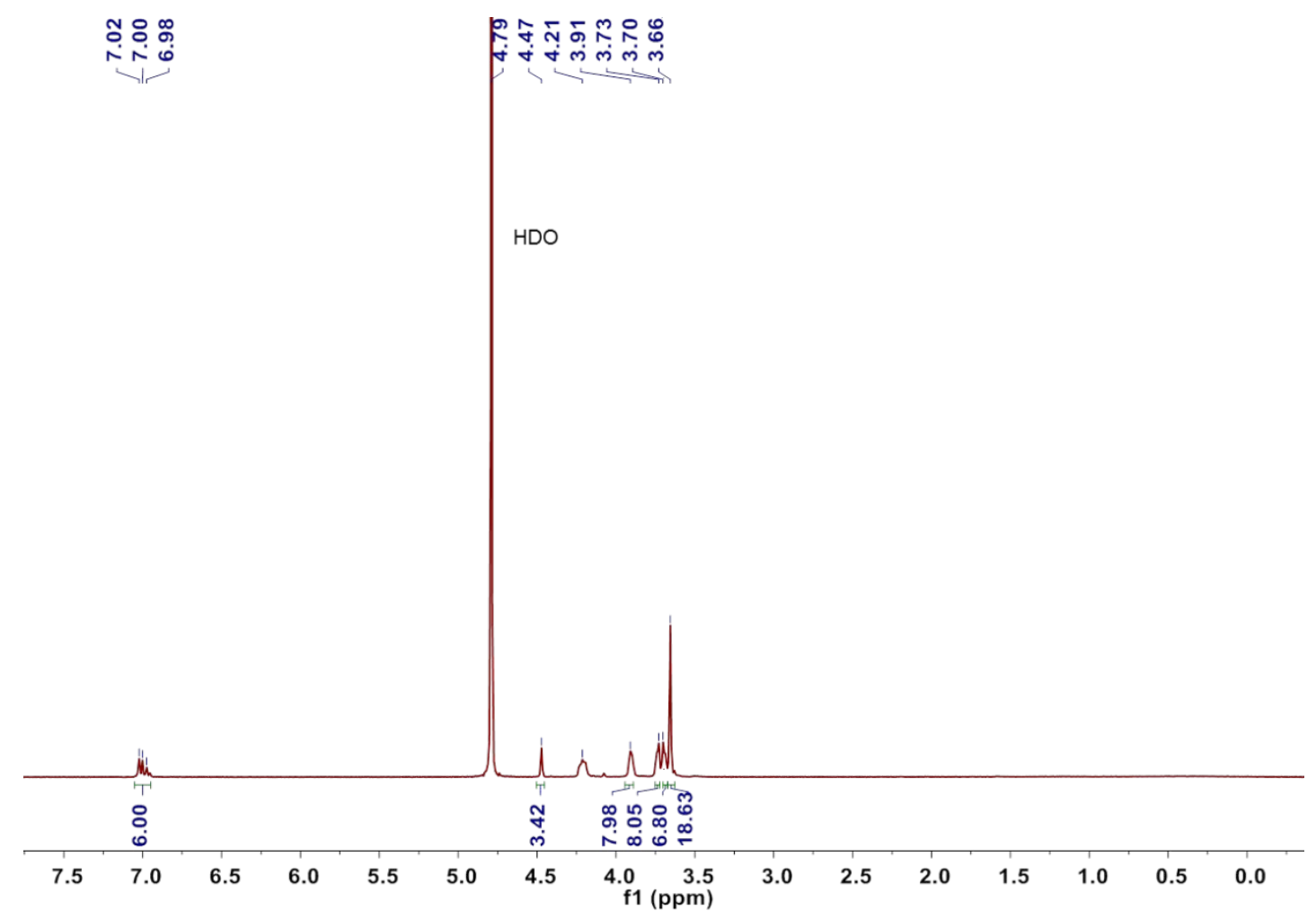

Figure S2. ${ }^{1} \mathrm{H}$ NMR spectrum $\left(400 \mathrm{MHz}, \mathrm{D}_{2} \mathrm{O}\right.$, room temperature) of $\mathbf{O F}$. OF shows poor solubility in water.

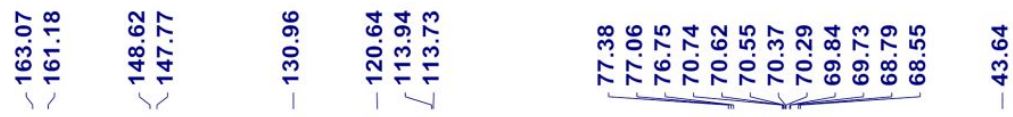

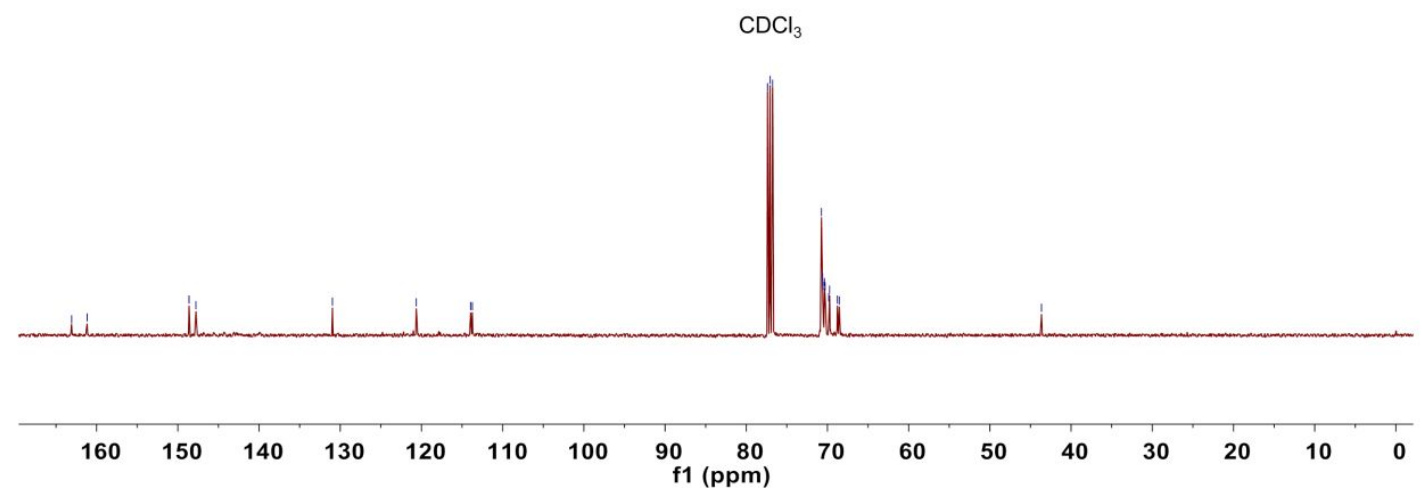

Figure S3. ${ }^{13} \mathrm{C}$ NMR spectrum $\left(100 \mathrm{MHz}, \mathrm{CDCl}_{3}\right.$, room temperature) of $\mathbf{O F}$. 
ণุ̣

ก

두윰ำ

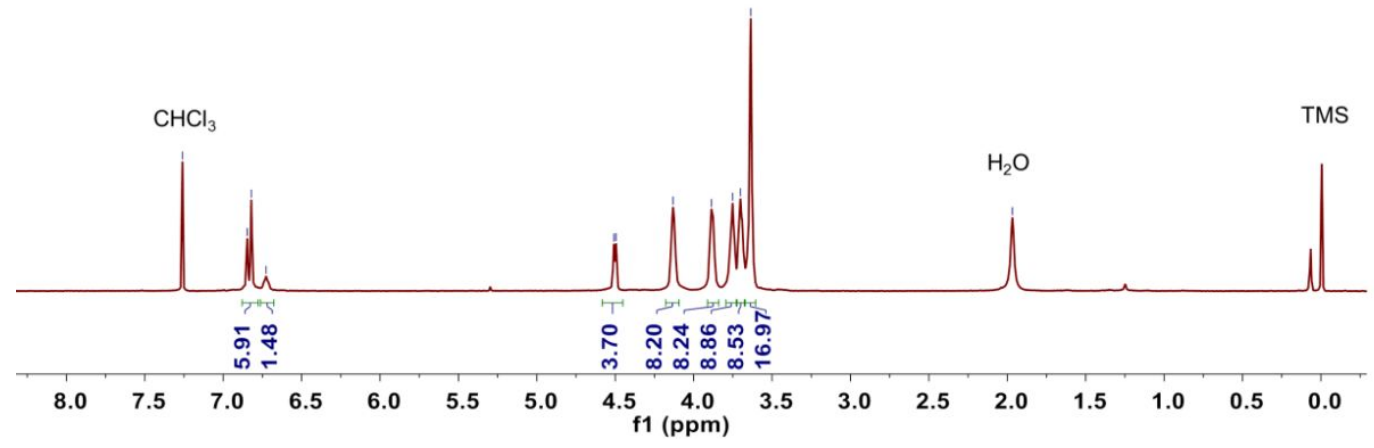

Figure S4. ${ }^{1} \mathrm{H}$ NMR spectrum (400 MHz, $\mathrm{CDCl}_{3}$, room temperature) of $\mathbf{M F}$.

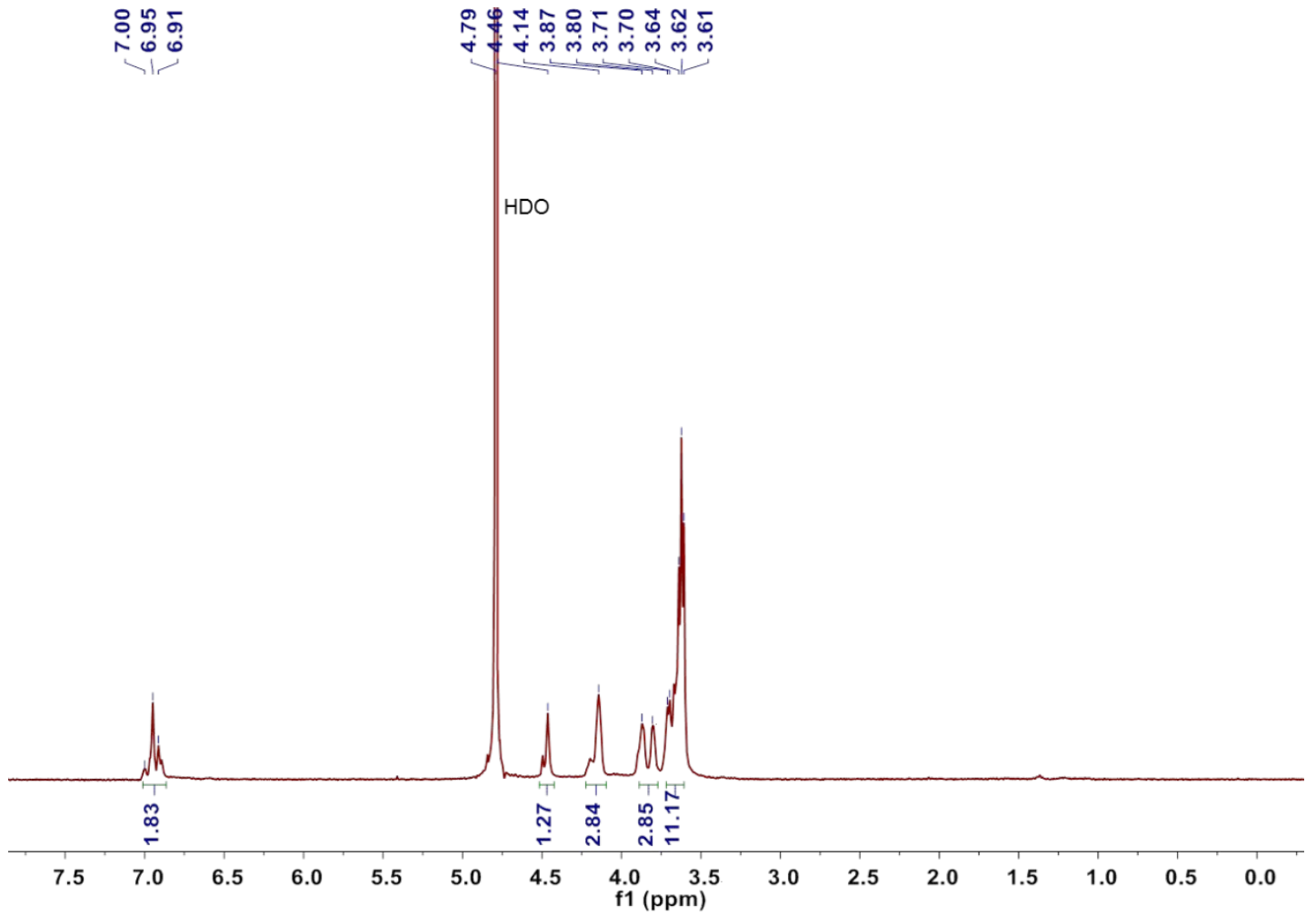

Figure S5. ${ }^{1} \mathrm{H}$ NMR spectrum (400 MHz, $\mathrm{D}_{2} \mathrm{O}$, room temperature) of MF. 


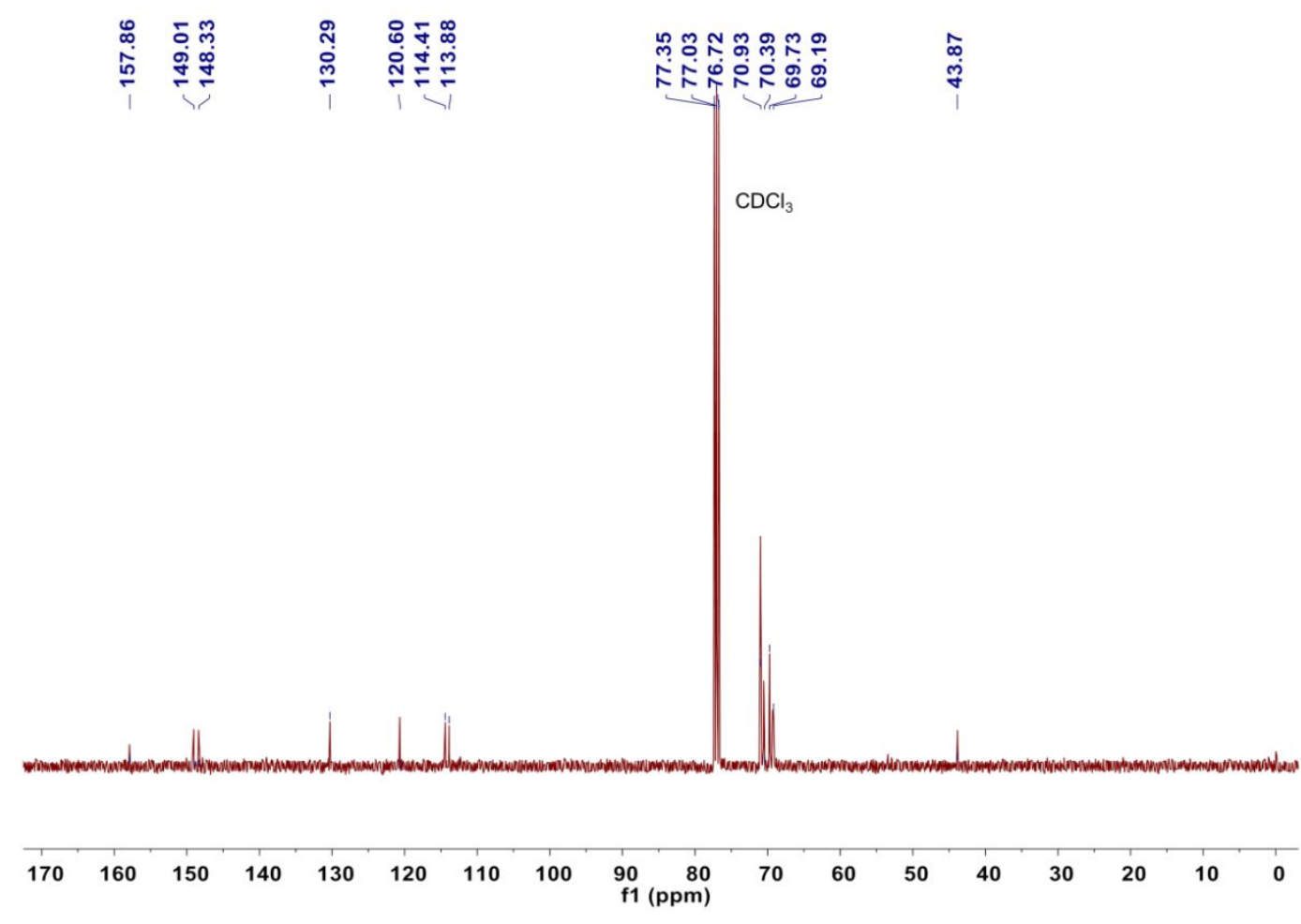

Figure S6. ${ }^{13} \mathrm{C}$ NMR spectrum (100 MHz, $\mathrm{CDCl}_{3}$, room temperature) of $\mathbf{M F}$.

\begin{tabular}{|c|c|}
\hline 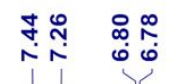 & 导邑 \\
\hline
\end{tabular}

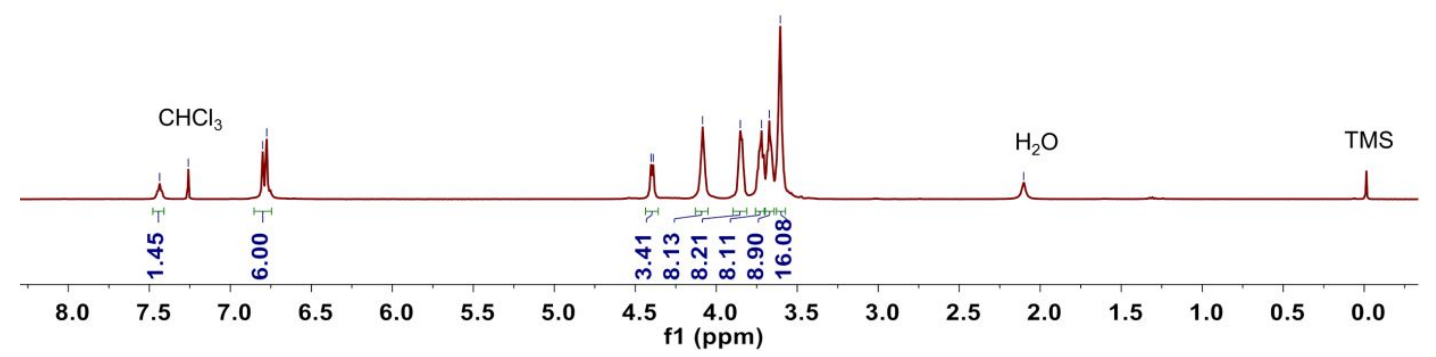

Figure S7. ${ }^{1} \mathrm{H}$ NMR spectrum (400 MHz, $\mathrm{CDCl}_{3}$, room temperature) of $\mathbf{P F}$. 


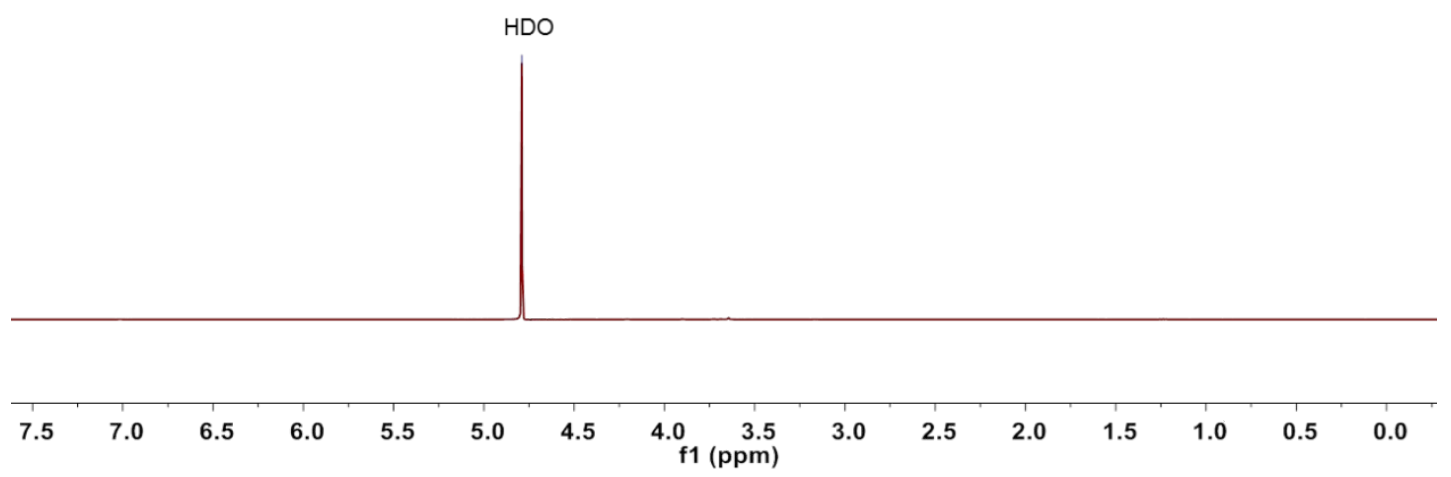

Figure S8. ${ }^{1} \mathrm{H}$ NMR spectrum (400 MHz, $\mathrm{D}_{2} \mathrm{O}$, room temperature) of PF. PF is insoluble in water.

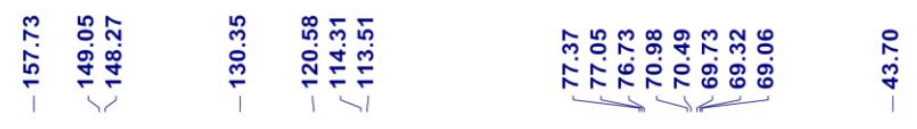

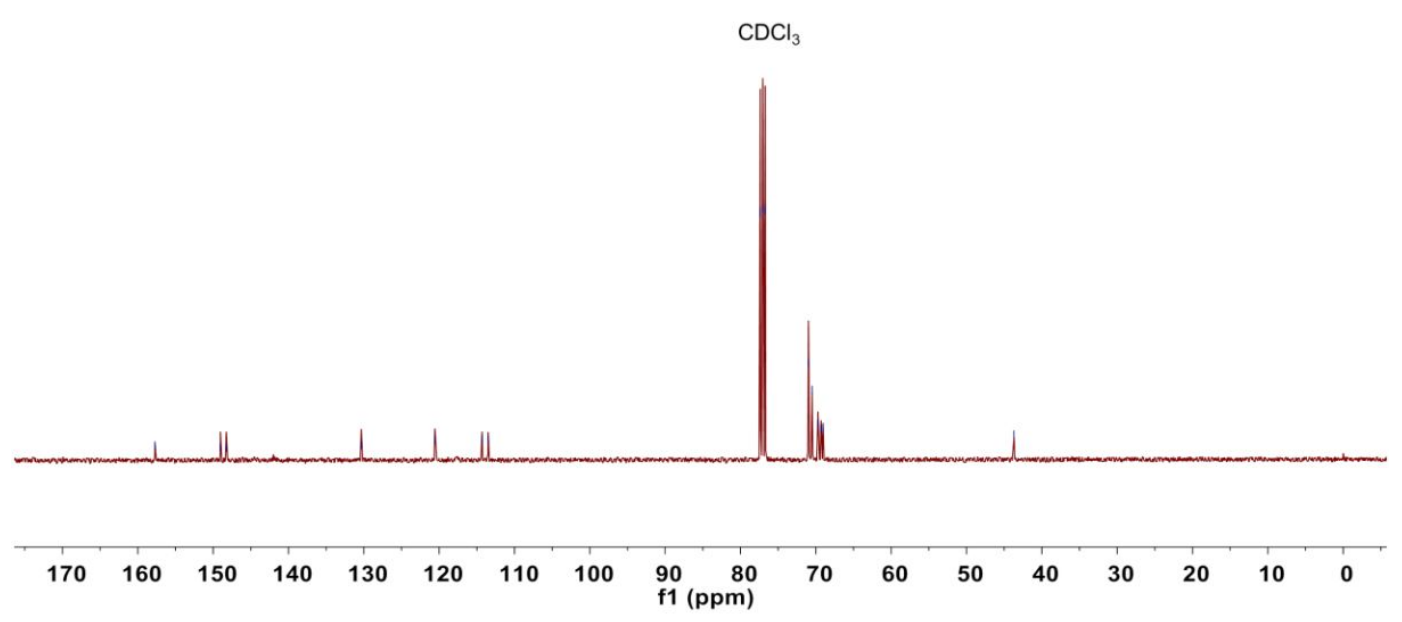

Figure S9. ${ }^{13} \mathrm{C}$ NMR spectrum $\left(100 \mathrm{MHz}, \mathrm{CDCl}_{3}\right.$, room temperature) of $\mathbf{P F}$. 
స

1
$\infty$

윰요

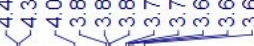
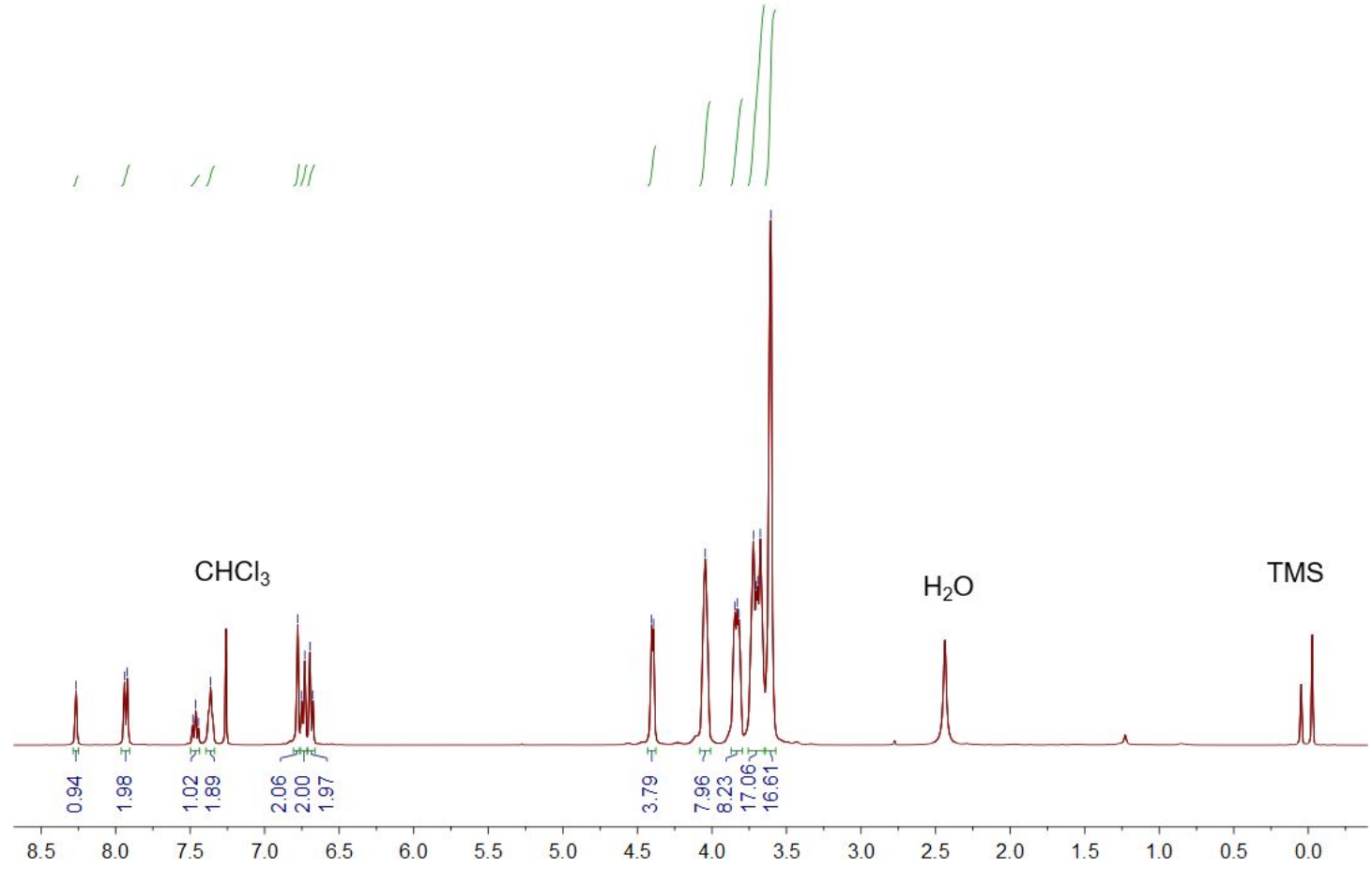

Figure S10. ${ }^{1} \mathrm{H}$ NMR spectrum $\left(400 \mathrm{MHz}, \mathrm{CDCl}_{3}\right.$, room temperature) of $\mathbf{M H}$.
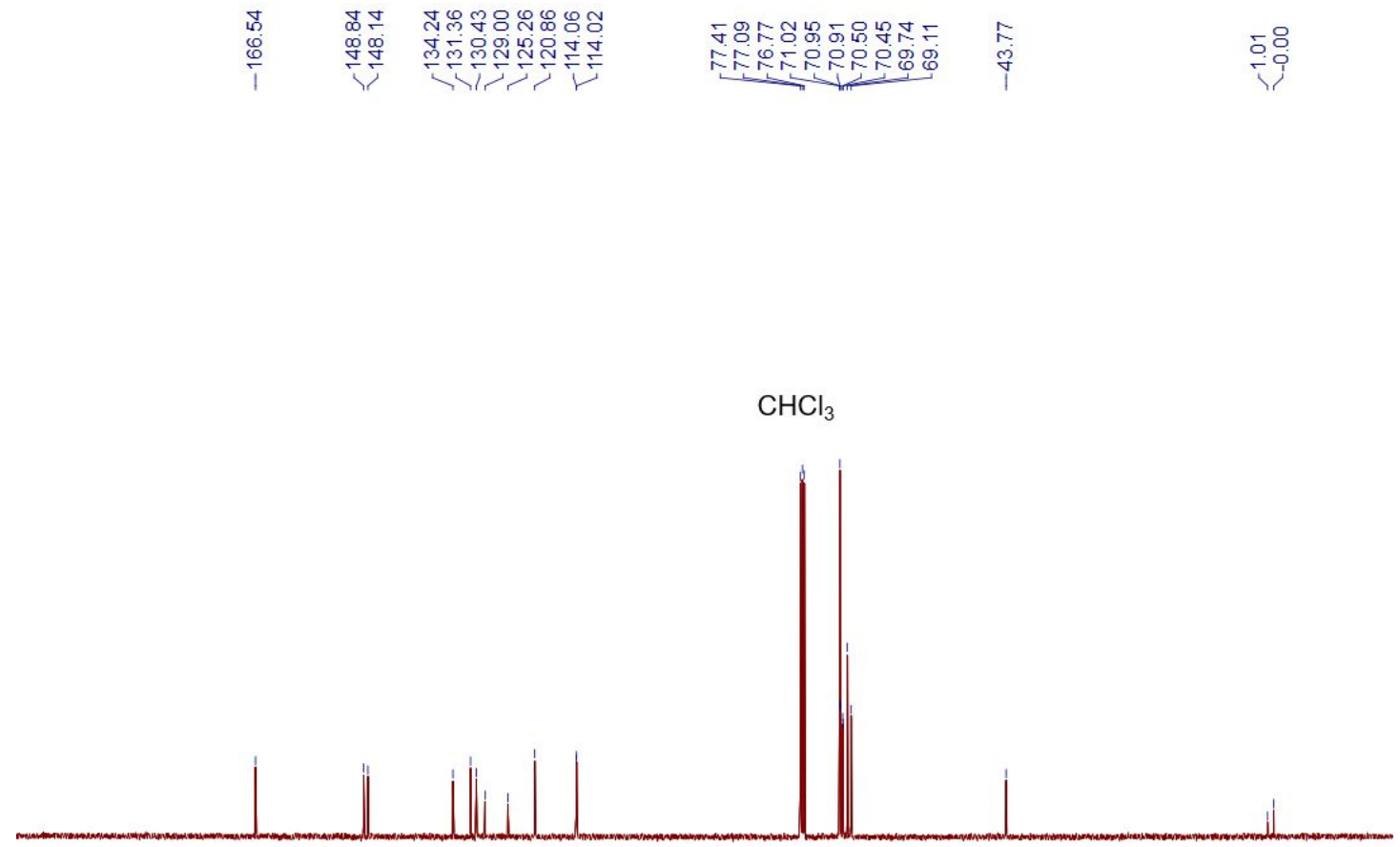

$\begin{array}{lllllllllllllllllllll}200 & 190 & 180 & 170 & 160 & 150 & 140 & 130 & 120 & 110 & 100 & 90 & 80 & 70 & 60 & 50 & 40 & 30 & 20 & 10 & 0\end{array}$

Figure S11. ${ }^{13} \mathrm{C}$ NMR spectrum $\left(100 \mathrm{MHz}, \mathrm{CDCl}_{3}\right.$, room temperature) of $\mathbf{M H}$. 


\section{Cycling tests of MF}

\begin{tabular}{cccccc}
\hline \multirow{2}{*}{ Cycle number } & \multicolumn{2}{c}{ Sol (transmittance value, \%) } & \multicolumn{2}{c}{ gel (transmittance value, \%) } \\
\cline { 2 - 3 } \cline { 5 - 6 } $10{ }^{\circ} \mathrm{C}$ & $30{ }^{\circ} \mathrm{C}$ & & $10{ }^{\circ} \mathrm{C}$ & $30{ }^{\circ} \mathrm{C}$ \\
\hline 1 & 94.7 & 1.9 & 98.6 & 4.2 \\
2 & 97.1 & 2.1 & 99.0 & 2.0 \\
3 & 97.1 & 2.5 & 98.3 & 2.0 \\
4 & 97.2 & 2.1 & 96.9 & 2.0 \\
5 & 98.0 & 1.9 & 98.0 & 1.8 \\
6 & 97.8 & 1.7 & 99.2 & 1.9 \\
\hline
\end{tabular}

Table S1: Transmittance value of $\mathbf{M F}(8.0 \mathrm{mg} / \mathrm{mL})$ at $550 \mathrm{~nm}$.

\section{Temperature-dependent $U V / V$ is of $M F$}
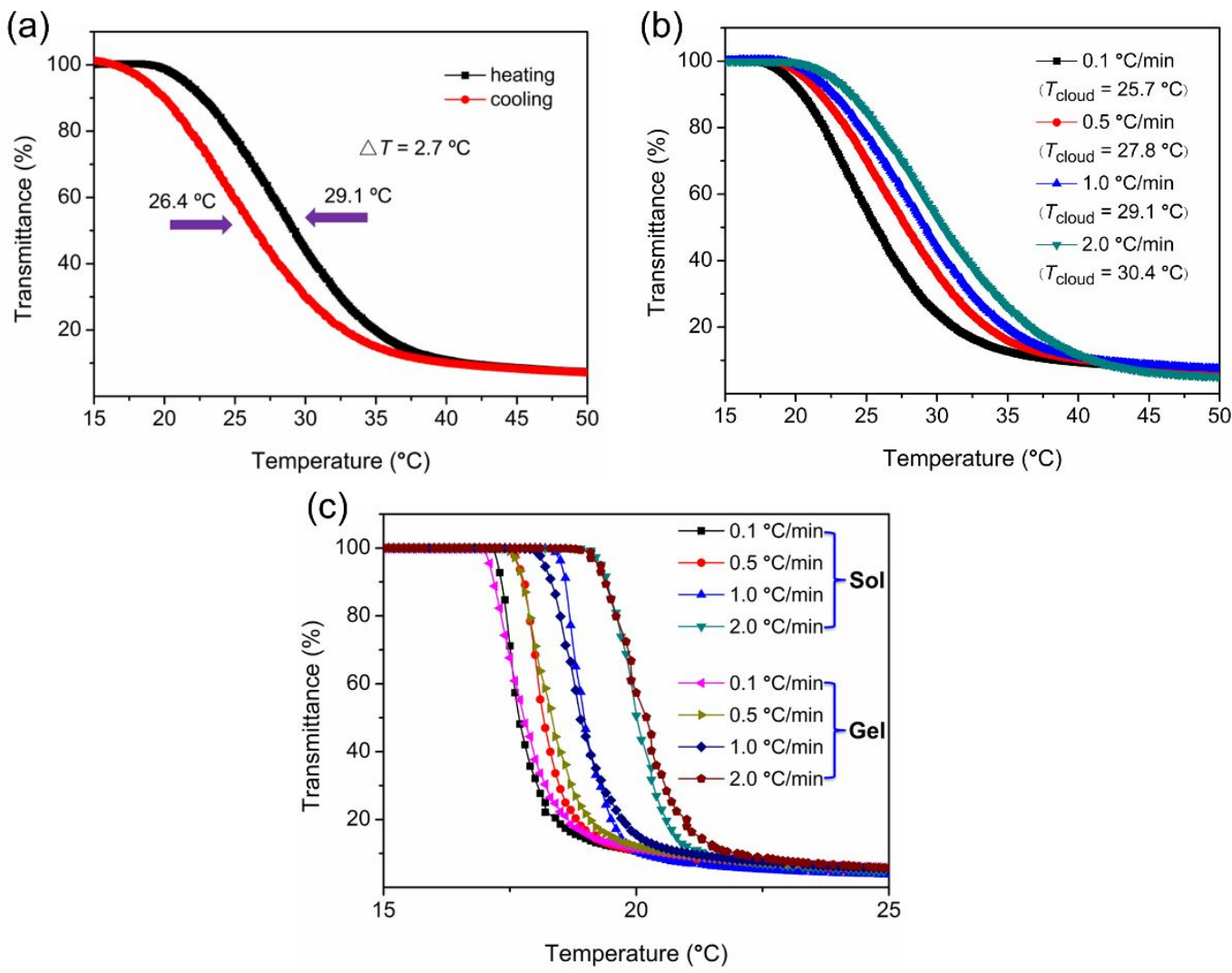

Figure S12. (a) Turbidity curves for MF $(3.0 \mathrm{mg} / \mathrm{mL})$ measured at $550 \mathrm{~nm}$. The rates for heating and cooling are $1.0{ }^{\circ} \mathrm{C} / \mathrm{min}$, respectively, (b) Turbidity curves for MF $(3.0 \mathrm{mg} / \mathrm{mL})$ measured at different heating rates, (c) heating-cooling cycles of $\mathbf{M F}(8.0 \mathrm{mg} / \mathrm{mL})$ at sol and gel states with different heating/cooling rates; 


\section{Rheology measurements of MF}
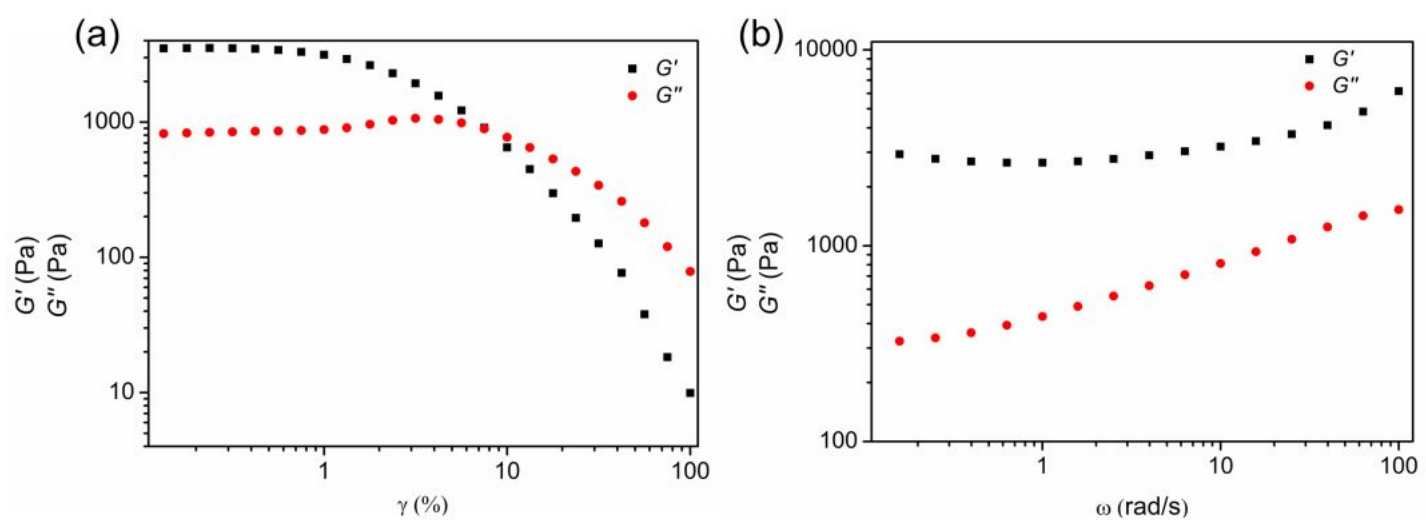

Figure S13. (a) $G$ ' and $G$ ' 'values of MF $(8.0 \mathrm{mg} / \mathrm{mL})$ on strain sweep and (b) $G$ ' and $G$ ', values of MF $(8.0 \mathrm{mg} / \mathrm{mL})$ on frequency sweep.

\section{Scanning electron microscopy of MF}
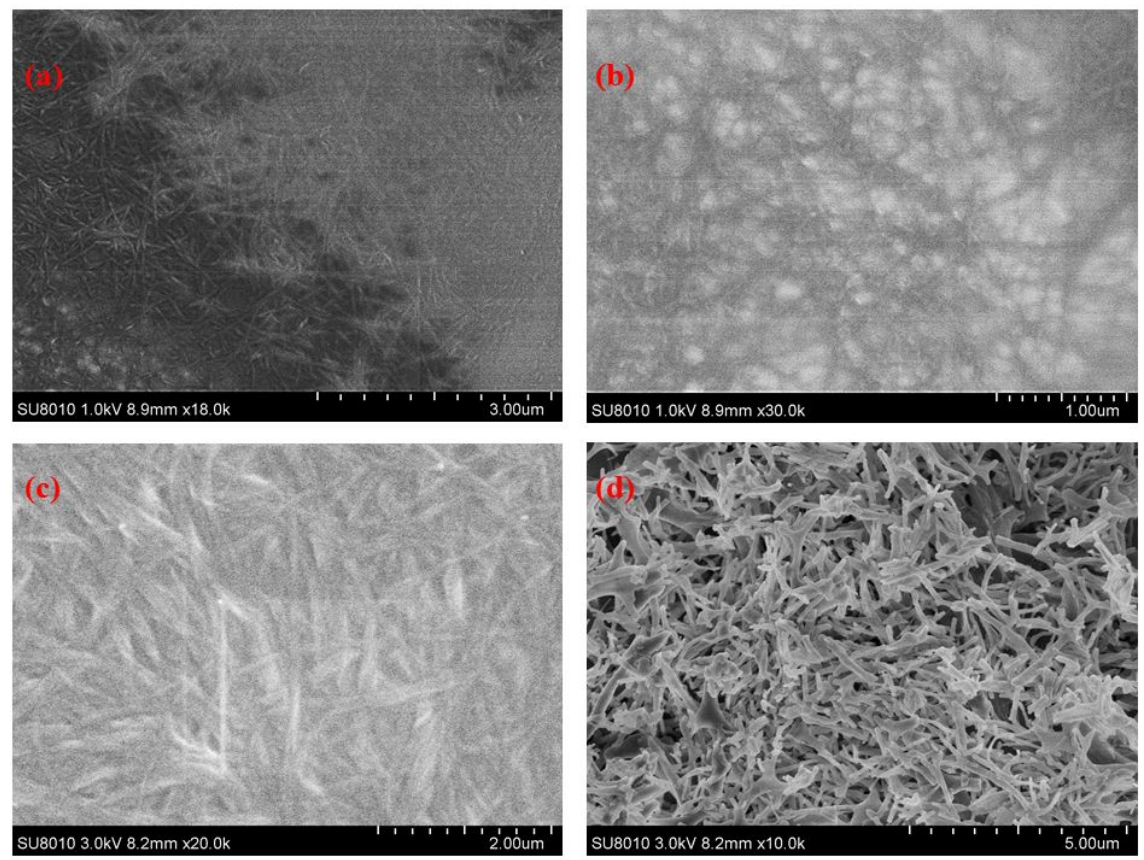

Figure S14. SEM images of xerogels MF at different concentrations: $(a, b)$ partial gels $(8.0$ $\mathrm{mg} / \mathrm{mL}$ ), (c) hydrogel $8.0 \mathrm{mg} / \mathrm{mL}$, (d) $4.0 \mathrm{mg} / \mathrm{mL}$.

As shown in Figure S14, typical fibers (assemble to networks) were observed from partial gelation or hydrogel MF samples. All samples were prepared by freeze-drying.

\section{Transmission electron microscopy of MF}



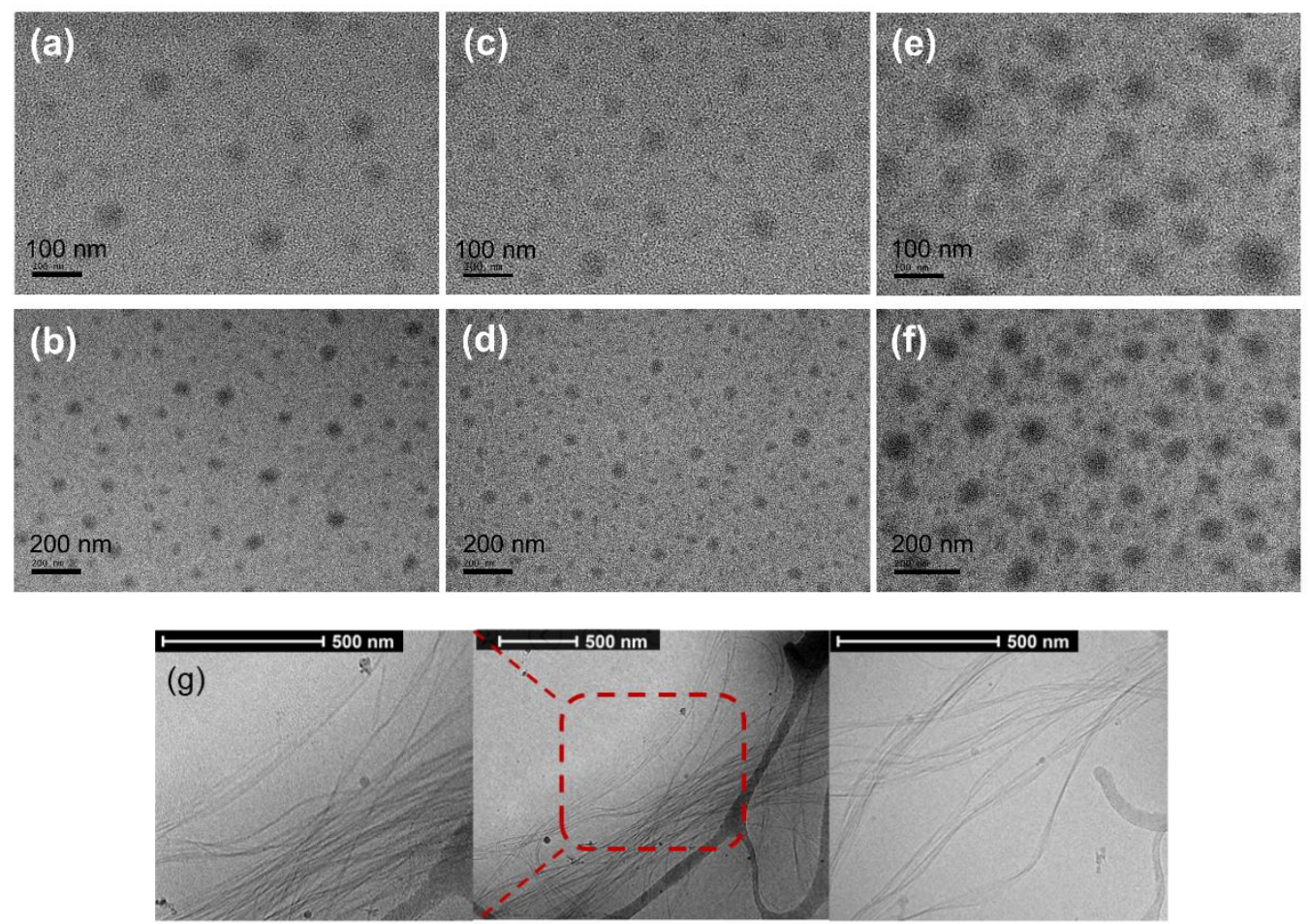

Figure S15. TEM images of MF at different concentrations: $(\mathrm{a}, \mathrm{b})$ are $1.0 \mathrm{mg} / \mathrm{mL} ;(\mathrm{c}, \mathrm{d})$ are $3.0 \mathrm{mg} / \mathrm{mL}$ and (e,f) are $8.0 \mathrm{mg} / \mathrm{mL}$. (g) Cryo-TEM images of MF above $T_{\text {cloud }}$ (sample was turbid during preparation).

In the TEM, due to the evaporation of solvents, monomers favor to form micelle-type aggregates (Figure S15a-f). Cryo-TEM measurements demonstrated that for MF samples above $T_{\text {cloud }}\left(29.7^{\circ} \mathrm{C}\right)$, long fibers $(\sim 1 \mu \mathrm{m}$ length, and 50-80 $\mathrm{nm}$ width) were observed (Figure $\mathrm{S} 15 \mathrm{~g})$. 


\section{Concentration-dependent ${ }^{1} \mathrm{H}$ NMR of MF}

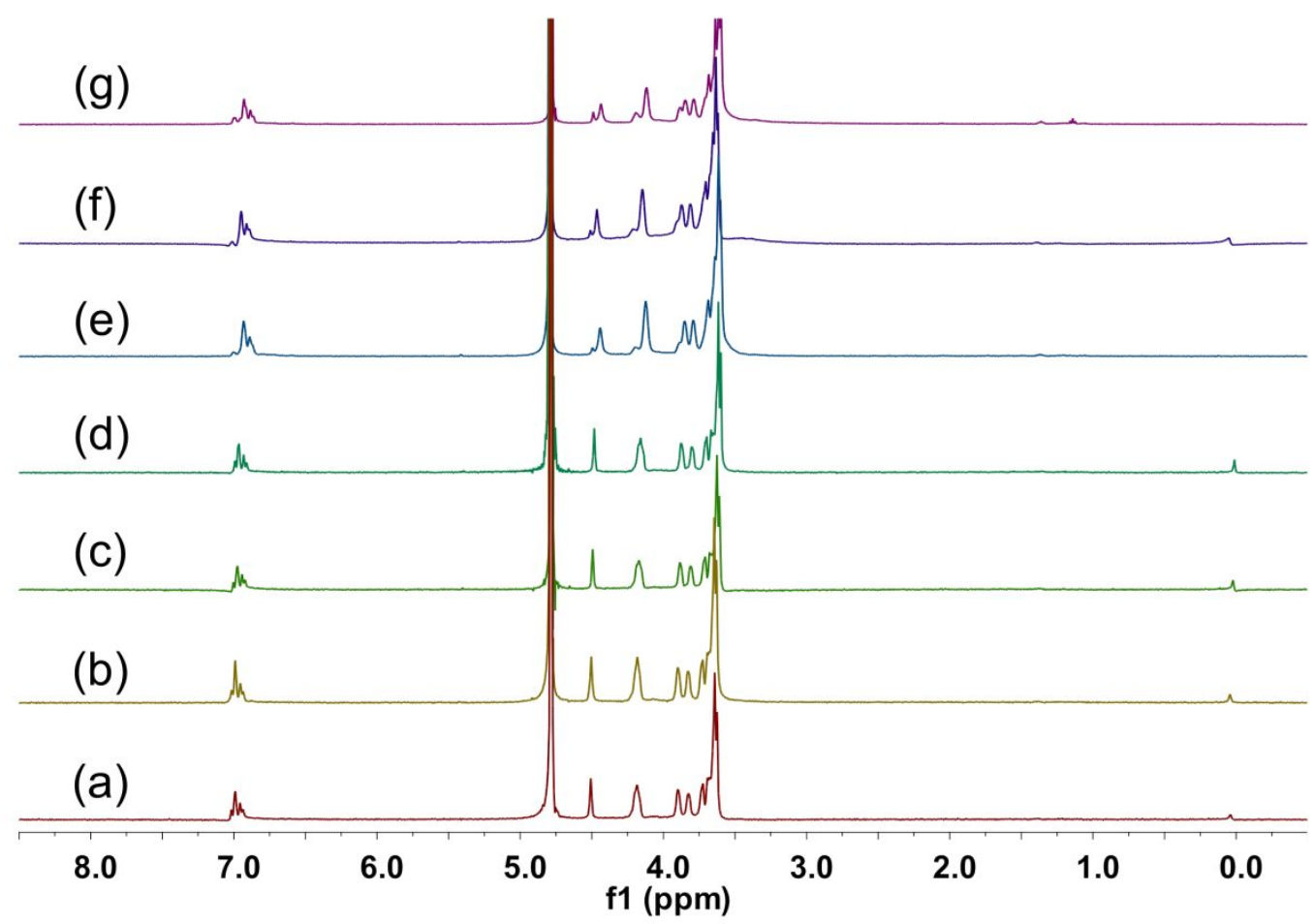

Figure S16. ${ }^{1} \mathrm{H}$ NMR spectra $\left(400 \mathrm{MHz}, \mathrm{D}_{2} \mathrm{O}, 25^{\circ} \mathrm{C}\right.$ ) of MF: (a) $1.4 \mathrm{mg} / \mathrm{mL}$, (b) $3.0 \mathrm{mg} / \mathrm{mL}$, (c) $4.0 \mathrm{mg} / \mathrm{mL}$, (d) $5.0 \mathrm{mg} / \mathrm{mL}$, (e) $6.0 \mathrm{mg} / \mathrm{mL}$, (f) $8.0 \mathrm{mg} / \mathrm{mL}$, and (f) $15 \mathrm{mg} / \mathrm{mL}$.

\section{Simulated chemical strucuture of MF}

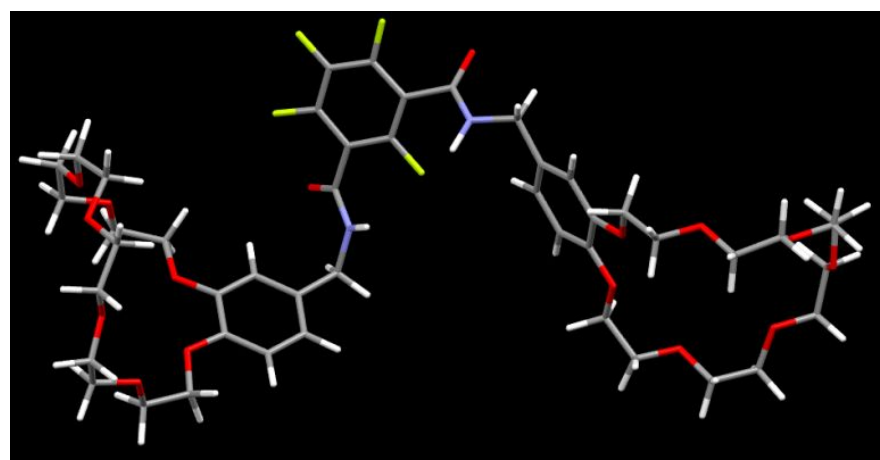

Figure S17. Simulated molecular structure of MF. 


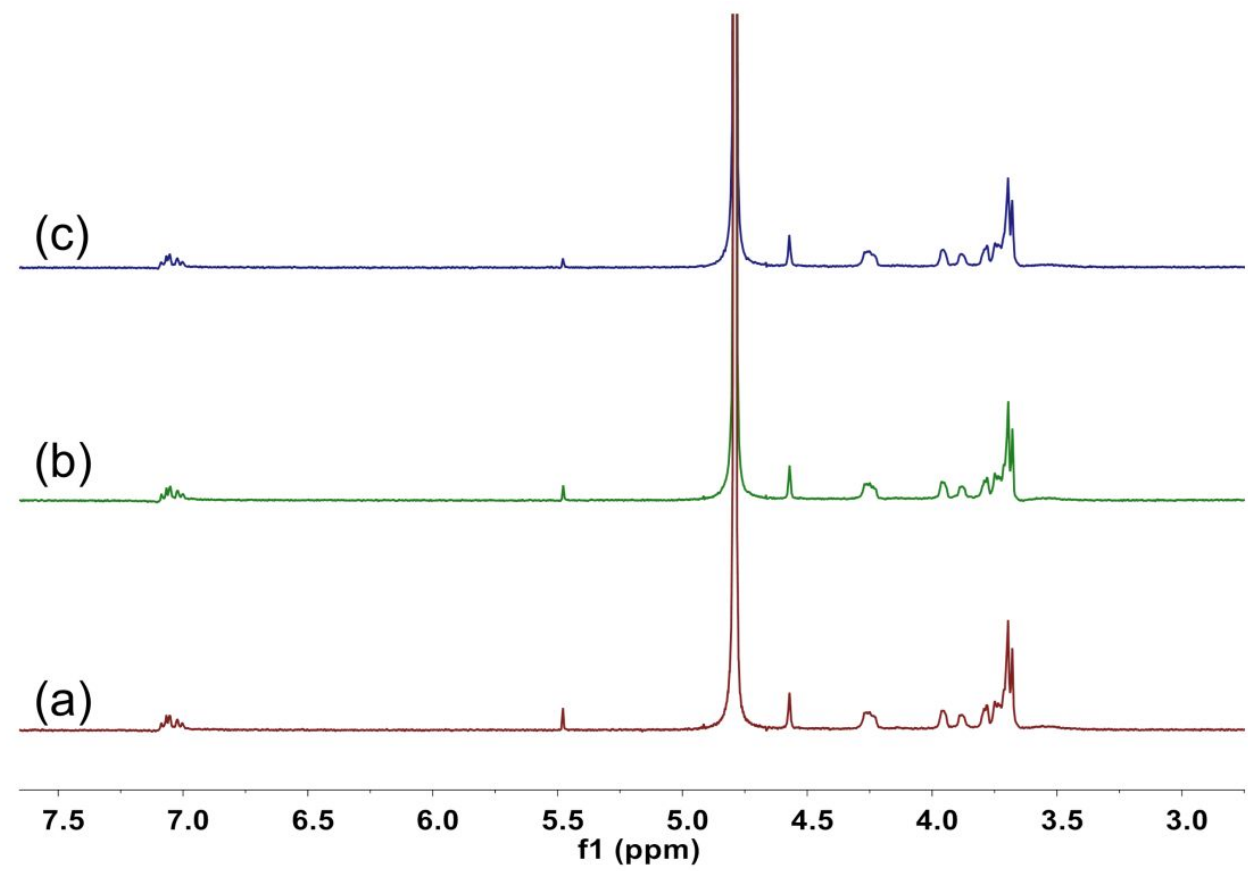

Figure S18. Time-dependent ${ }^{1} \mathrm{H}$ NMR spectra $\left(400 \mathrm{MHz}, \mathrm{D}_{2} \mathrm{O}, 25{ }^{\circ} \mathrm{C}\right)$ of $\mathbf{M F}(1.0 \mathrm{mg} / \mathrm{mL})$ : (a) $0 \mathrm{~h}$, (b) $48 \mathrm{~h}$, (c) $96 \mathrm{~h}$. No changes were observed in the time-dependent ${ }^{1} \mathrm{H}$ NMR tests.

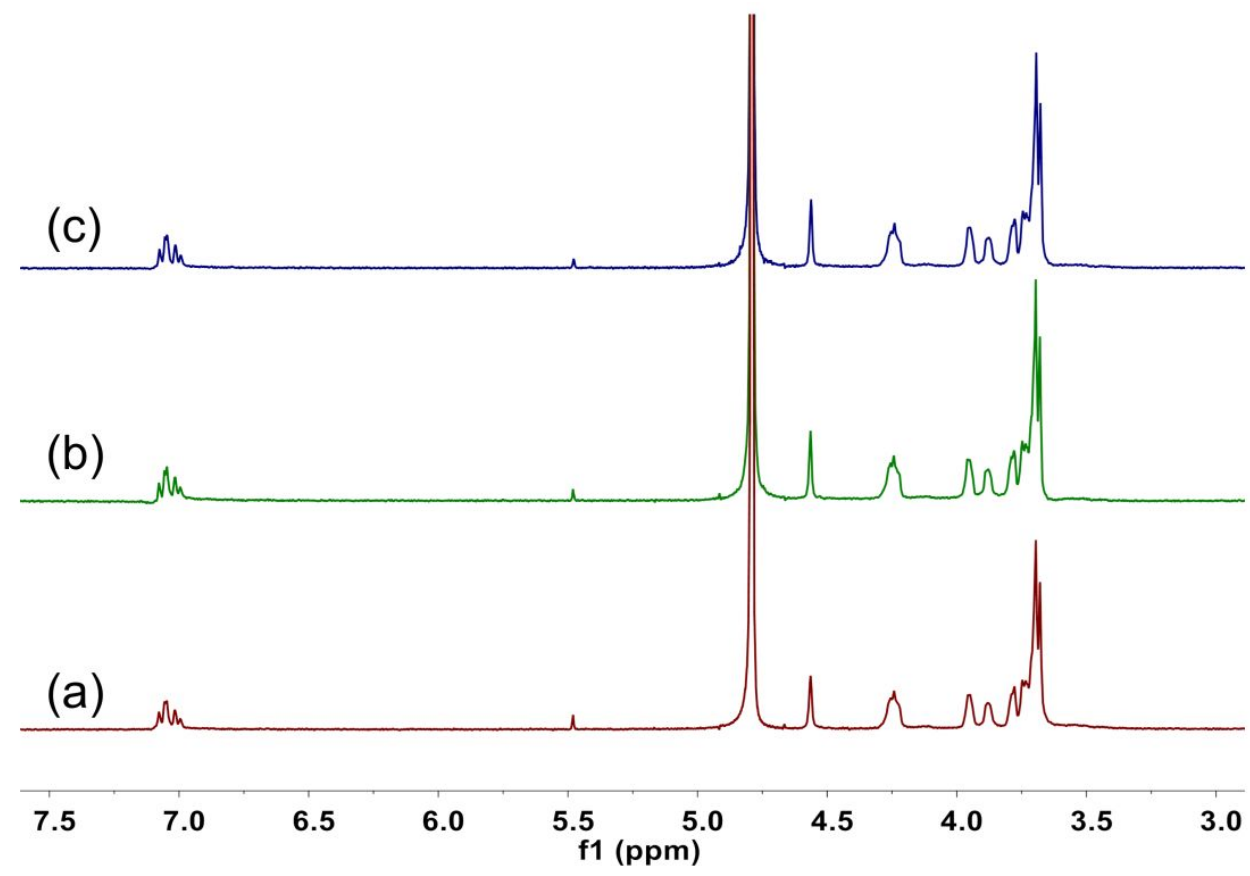

Figure S19. Time-dependent ${ }^{1} \mathrm{H}$ NMR spectra $\left(400 \mathrm{MHz}, \mathrm{D}_{2} \mathrm{O}, 25^{\circ} \mathrm{C}\right)$ of $\mathbf{M F}(3.0 \mathrm{mg} / \mathrm{mL})$ : (a) $0 \mathrm{~h}$, (b) $48 \mathrm{~h}$, (c) $96 \mathrm{~h}$. No changes were observed in the time-dependent ${ }^{1} \mathrm{H}$ NMR tests. 


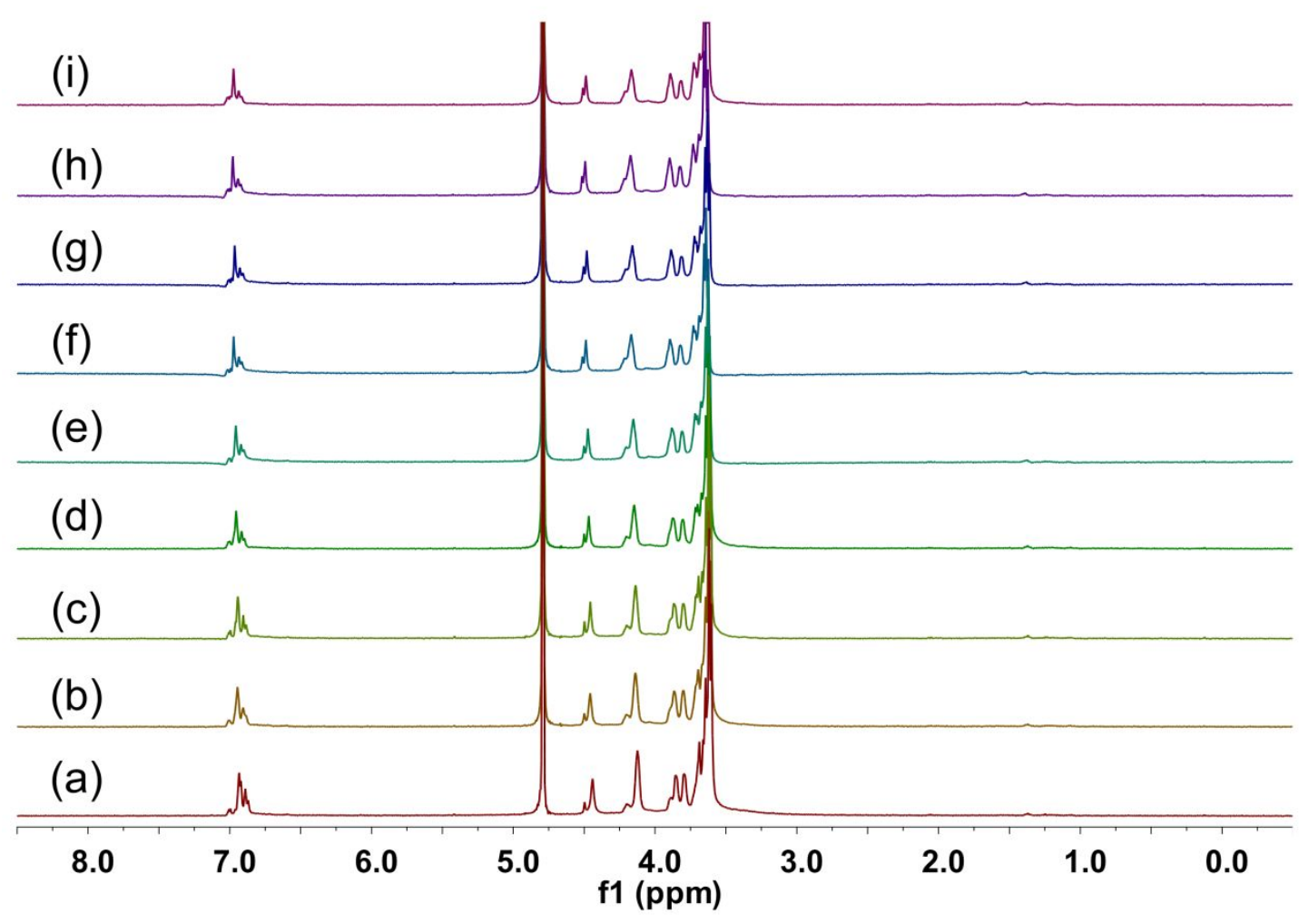

Figure S20. Time-dependent ${ }^{1} \mathrm{H}$ NMR spectra $\left(400 \mathrm{MHz}, \mathrm{D}_{2} \mathrm{O}, 25^{\circ} \mathrm{C}\right)$ of $\mathbf{M F}(8.0 \mathrm{mg} / \mathrm{mL})$ : (a) $0 \mathrm{~h}$, (b) $2 \mathrm{~h}$, (c) $3 \mathrm{~h}$, (d) $5 \mathrm{~h}$, (e) $9 \mathrm{~h}$, (f) $21 \mathrm{~h}$, (g) $33 \mathrm{~h}$, (h) $54 \mathrm{~h}$, (i) $82 \mathrm{~h}$.

\section{Temperature-dependent ${ }^{1} \mathrm{H}$ NMR of MF}

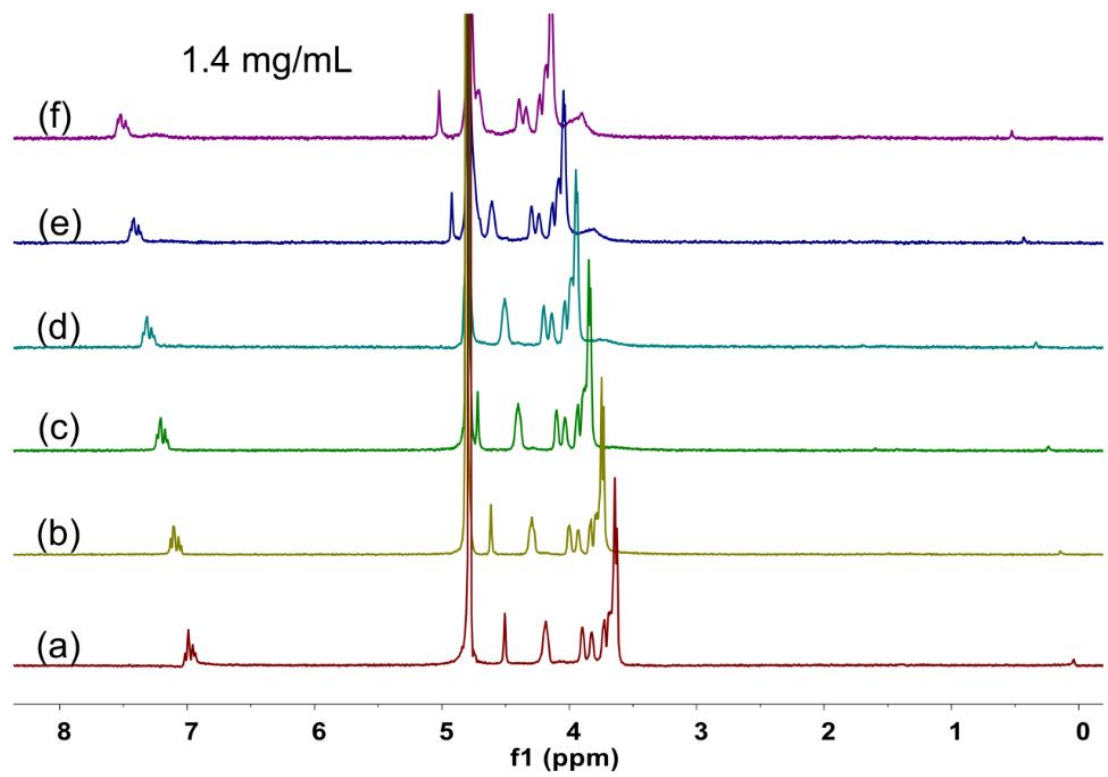

Figure S21. Temperature-dependent ${ }^{1} \mathrm{H}$ NMR spectra (400 MHz, $\mathrm{D}_{2} \mathrm{O}$ ) of $\mathbf{M F}$ : (a) $25^{\circ} \mathrm{C}$, (b) $30{ }^{\circ} \mathrm{C}$, (c) $40^{\circ} \mathrm{C}$, (d) $50{ }^{\circ} \mathrm{C}$, (e) $60^{\circ} \mathrm{C}$, (f) $70^{\circ} \mathrm{C}$. 


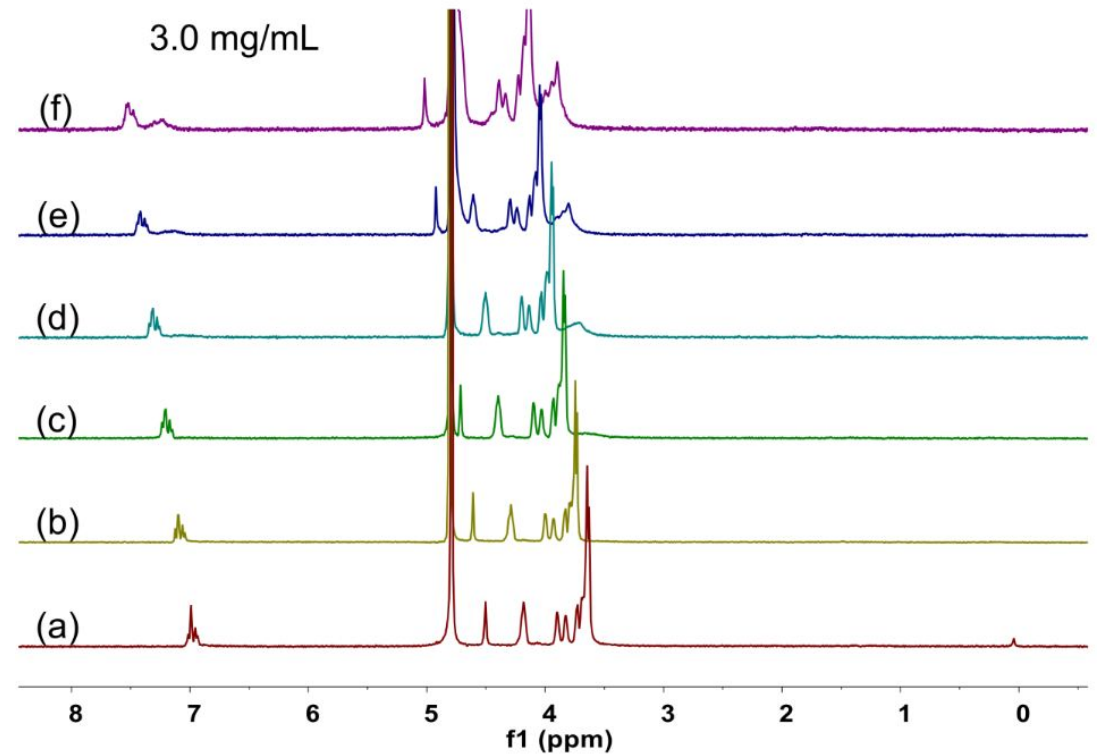

Figure S22. Temperature-dependent ${ }^{1} \mathrm{H}$ NMR spectra (400 MHz, $\mathrm{D}_{2} \mathrm{O}$ ) of MF: (a) $25^{\circ} \mathrm{C}$, (b) $30{ }^{\circ} \mathrm{C}$, (c) $40^{\circ} \mathrm{C}$, (d) $50{ }^{\circ} \mathrm{C}$, (e) $60^{\circ} \mathrm{C}$, (f) $70^{\circ} \mathrm{C}$.

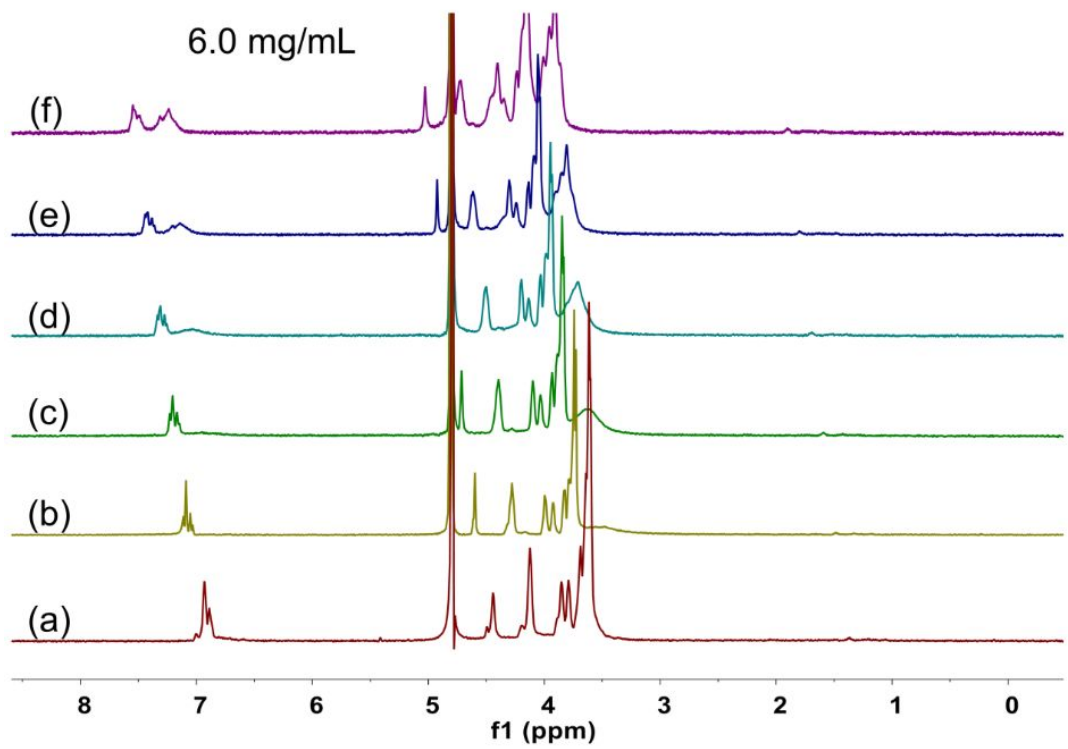

Figure S23. Temperature-dependent ${ }^{1} \mathrm{H}$ NMR spectra (400 MHz, $\mathrm{D}_{2} \mathrm{O}$ ) of MF: (a) $25^{\circ} \mathrm{C}$, (b) $30{ }^{\circ} \mathrm{C}$, (c) $40^{\circ} \mathrm{C}$, (d) $50{ }^{\circ} \mathrm{C}$, (e) $60^{\circ} \mathrm{C}$, (f) $70^{\circ} \mathrm{C}$. 


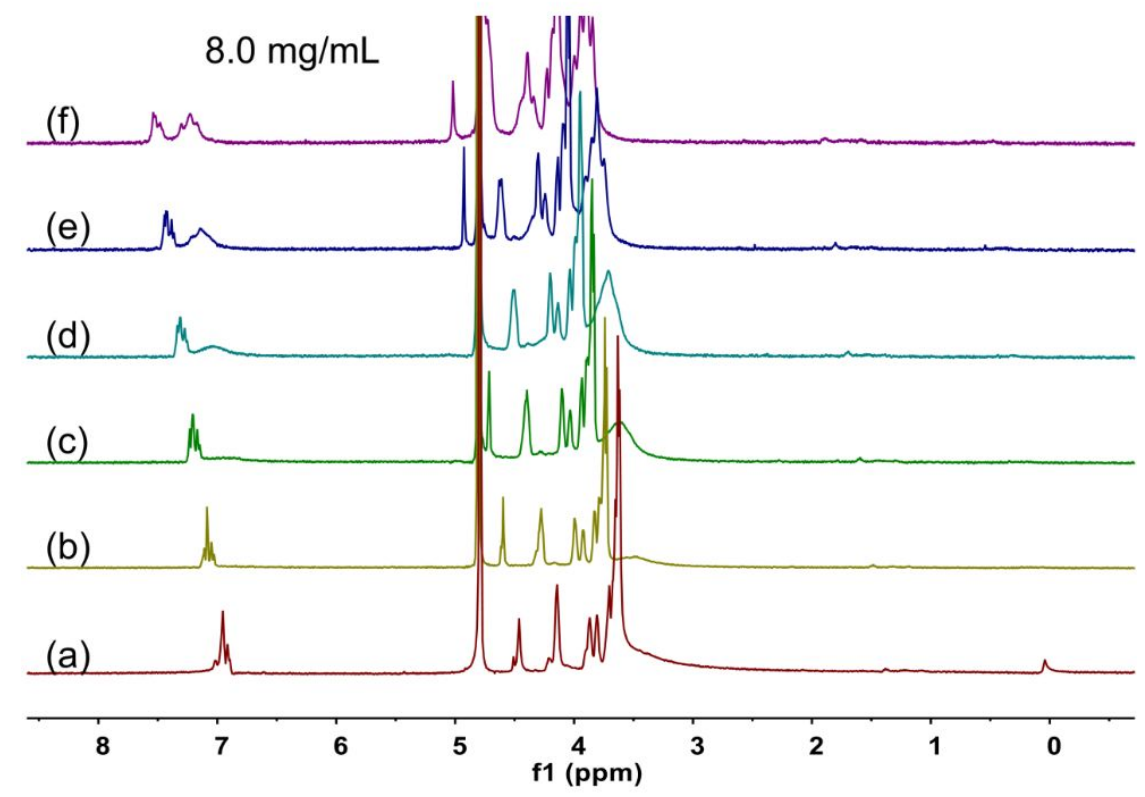

Figure S24. Temperature-dependent ${ }^{1} \mathrm{H}$ NMR spectra (400 MHz, $\mathrm{D}_{2} \mathrm{O}$ ) of MF: (a) $25^{\circ} \mathrm{C}$, (b) $30{ }^{\circ} \mathrm{C}$, (c) $40^{\circ} \mathrm{C}$, (d) $50{ }^{\circ} \mathrm{C}$, (e) $60^{\circ} \mathrm{C}$, (f) $70^{\circ} \mathrm{C}$.

\section{Time-/concentration-/temperature-dependent ${ }^{1} \mathrm{H}$ NMR of MH}

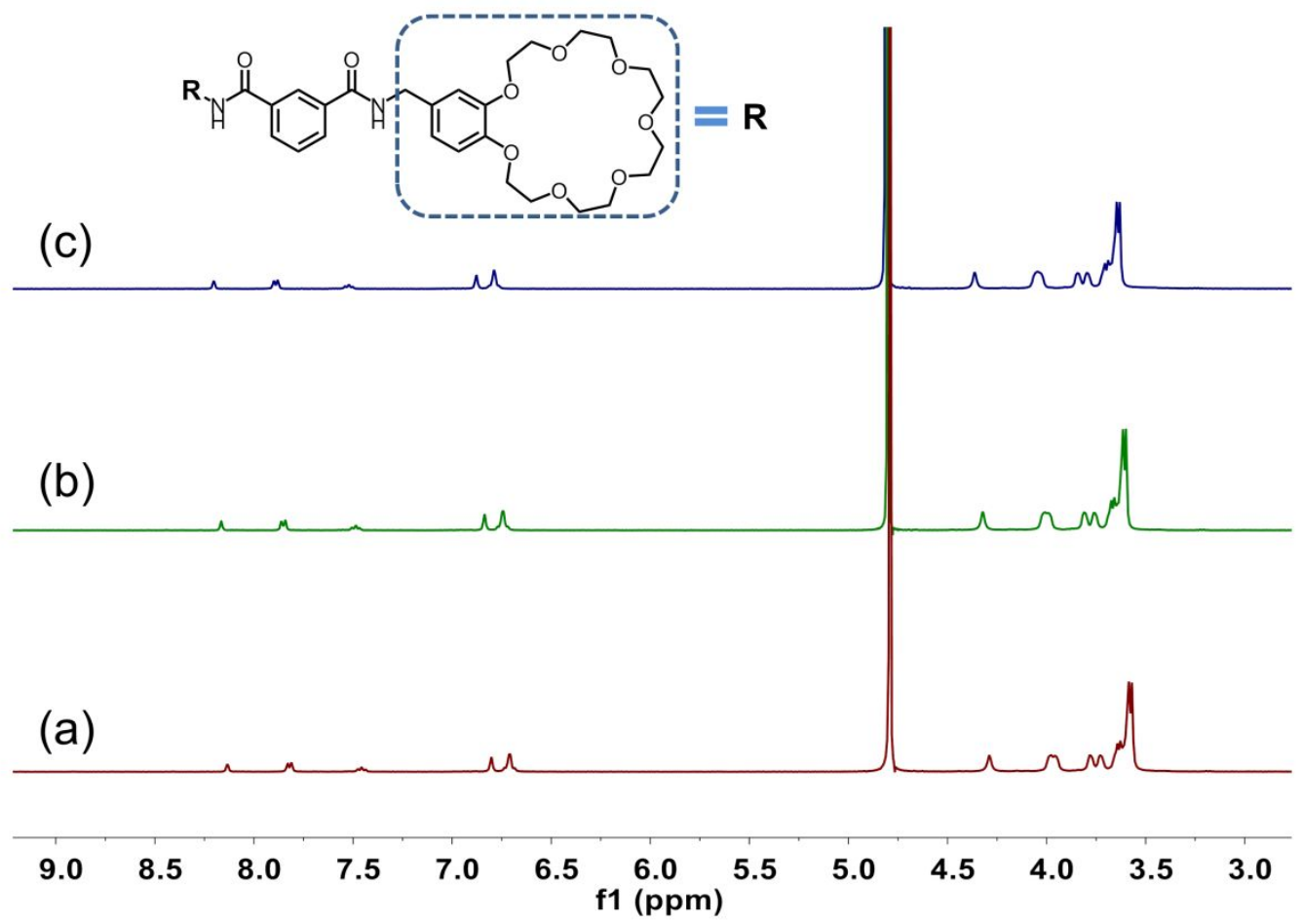

Figure S25. Time-dependent ${ }^{1} \mathrm{H}$ NMR spectra of $\mathbf{M H}\left(400 \mathrm{MHz}, \mathrm{D}_{2} \mathrm{O}, 25{ }^{\circ} \mathrm{C}\right)$ of $(8.0$ $\mathrm{mg} / \mathrm{mL}$ ): (a) $0 \mathrm{~h}$, (b) $24 \mathrm{~h}$, (c) $240 \mathrm{~h}$. No changes were observed in the time-dependent ${ }^{1} \mathrm{H}$ NMR tests. 


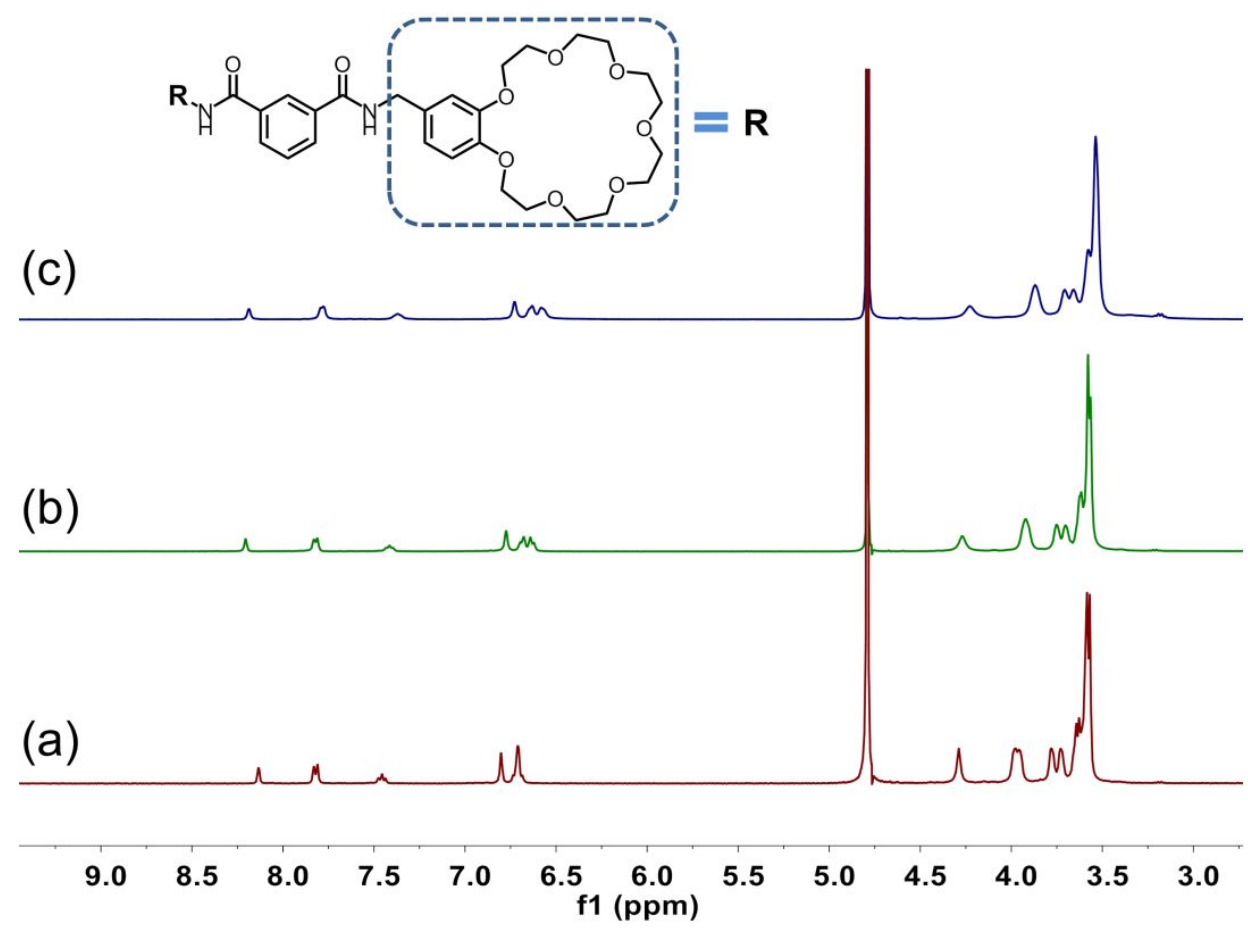

Figure S26. ${ }^{1} \mathrm{H}$ NMR spectra (400 MHz, $\mathrm{D}_{2} \mathrm{O}, 25^{\circ} \mathrm{C}$ ) of MH: (a) $8.0 \mathrm{mg} / \mathrm{mL}$, (b) $16 \mathrm{mg} / \mathrm{mL}$, (c) $90 \mathrm{mg} / \mathrm{mL}$.

(g)

(f)

(e)

(d)

(c)

(b)

(a)

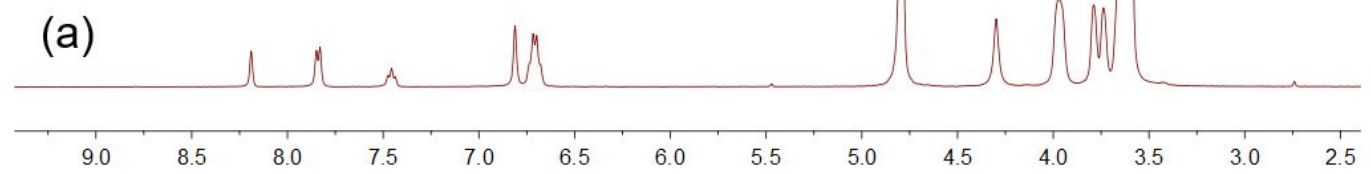

Figure S27. Temperature-dependent ${ }^{1} \mathrm{H}$ NMR spectra $\left(400 \mathrm{MHz}, \mathrm{D}_{2} \mathrm{O}, 8.0 \mathrm{mg} / \mathrm{mL}\right)$ of $\mathbf{M H}$ : (a) $25^{\circ} \mathrm{C}$, (b) $30^{\circ} \mathrm{C}$, (c) $35^{\circ} \mathrm{C}$, (d) $40{ }^{\circ} \mathrm{C}$, (e) $50{ }^{\circ} \mathrm{C}$, (f) $60^{\circ} \mathrm{C}$, (g) $70{ }^{\circ} \mathrm{C}$. 


\section{Concentration of the sol part of MF hydrogel}

When the hydrogel $\left(8.0 \mathrm{mg} / \mathrm{mL}\right.$, turbid) was placed above $T_{\text {cloud, }}$, a small amount of sol was slowly isolated from the gel. The concentration of sol $(4.0 \mathrm{mg} / \mathrm{ml})$ was determined by solvent evaporation method.

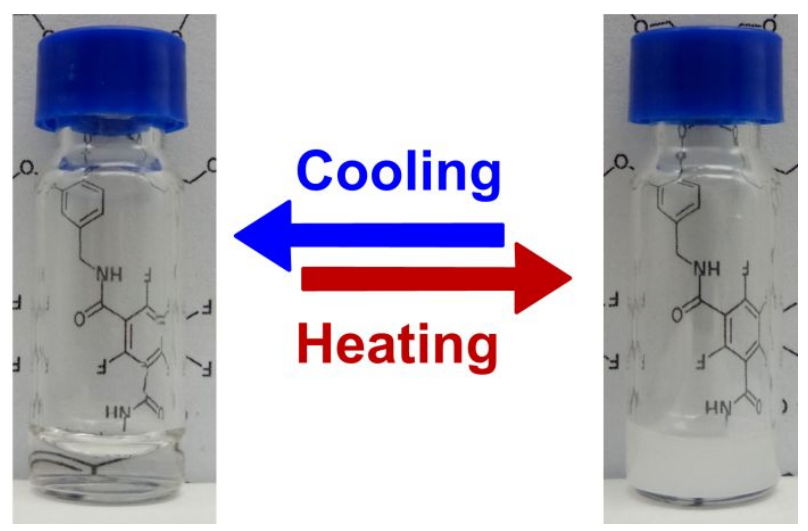

Figure S28. Photographs of the sol isolated from the gel of MF: clear sol under cooling (left), turbid sol under heating (right).

\section{Reference}

[1] a) Qi, Z., de Molina, P. M., Jiang, W., Wang, Q., Nowosinski, K., Schulz, A., Gradzielski, M., Schalley, C. A. Systems chemistry: Logic gates based on the stimuli-responsive gel-sol transition of a crown ether-functionalized bis(urea) gelator. Chem. Sci., 2012, 3, 2073-2082. b) Dong, S; Leng, J.; Feng, Y.; Liu, M.; Stackhouse, C. J.; Schonhals, A; Chiappisi, L.; Gao, L.; Chen, W.; Shang, J.; Jin, L.; Qi, Z.; Schalley, C. A. Structural water as an essential comonomer in supramolecular polymerization. Sci. Adv., 2017, 3, eaao0900. 